\title{
Premotor Ramping of Thalamic Neuronal Activity Is Modulated by Nigral Inputs and Contributes to Control the Timing of Action Release
}

\author{
${ }^{\circledR}$ Julien Catanese and ${ }^{\circledR}$ Dieter Jaeger \\ Department of Biology, Emory University, Atlanta, Georgia 30322
}

The ventromedial $(\mathrm{VM}) /$ ventro-anterior-lateral $(\mathrm{VAL})$ motor thalamus is a key junction within the brain circuits sustaining normal and pathologic motor control functions and decision-making. In this area of thalamus, on one hand, the inhibitory nigro-thalamic pathway provides a main output from the basal ganglia, and, on the other hand, motor thalamo-cortical loops are involved in the maintenance of ramping preparatory activity before goal-directed movements. To better understand the nigral impact on thalamic activity, we recorded electrophysiological responses from VM/VAL neurons while male and female mice were performing a delayed right/left decision licking task. Analysis of correct (corr) and error trials revealed that thalamic ramping activity was stronger for premature licks (impulsive action) and weaker for trials with no licks [omission (omi)] compared with correct trials. Suppressing ramping activity through optogenetic activation of nigral terminals in the motor thalamus during the delay epoch of the task led to a reduced probability of impulsive action and an increased amount of omissions trials. We propose a parsimonious model explaining our data and conclude that a thalamic ramping mechanism contributes to the control of proper timing of action release and that inhibitory nigral inputs are sufficient to interrupt this mechanism and modulate the amount of motor impulsivity in this task.

Key words: basal ganglia; behavior; electrophysiology; mouse; optogenetics; VM thalamus

\section{Significance Statement}

Coordinated neural activity in motor circuits is essential for correct movement preparation and execution, and even slight imbalances in neural processing can lead to failure in behavioral tasks or motor disorders. Here we focused on how failure to regulate the control of activity balance in the motor thalamus can be implicated in impulsive action release or omissions to act, through an activity ramping mechanism that is required for proper action release. Using optogenetic activation of inhibitory basal ganglia terminals in motor thalamus we show that basal ganglia input is well positioned to control this ramping activity and determine the timing of action initiation.

\section{Introduction}

The basal ganglia (BG) have been implicated in action selection (Mink, 1996; Donahue and Kreitzer, 2015) and motor learning (Graybiel, 2008), and in the control of movement vigor (Turner

Received May 15, 2020; revised Nov. 8, 2020; accepted Dec. 28, 2020.

Author contributions: J.C. and D.J. designed research; J.C. performed research; J.C. analyzed data; J.C. and D.J. wrote the paper.

This work was supported by National Institutes of Health Grants 1U01-NS-094302 [to D.J., Principal Investigator (PI)], P50-NS-098685 (to D.J., PI), and 1R01-NS-111470 (to D.J., PI) under the BRAIN Initiative. We thank Dr. Adriana Galvan for help with histological procedures, Lowell Ramsey for constructing the lick sensor circuit, Dr. Nick Steinmetz for help with 3D electrode track analysis, and Dr. Hidehiko Inagaki for insightful comments on the draft. We also thank members of the Jaeger laboratory, Dr. Li Su, Dr. Arthur Morissette, Chelsea Leversedge, and Jacqueline Zhu for technical help.

The authors declare no competing financial interests.

Correspondence should be addressed to Dieter Jaeger at djaeger@emory.edu or Julien Catanese at catanese.julien@gmail.com.

https://doi.org/10.1523/JNEUROSCI.1204-20.2020

Copyright $\odot 2021$ the authors and Desmurget, 2010). In addition, neuronal mechanisms that control the timing of movement are critical for proper behavior and may involve the basal ganglia (Buhusi and Meck, 2005). The classic conceptual model of basal ganglia circuitry posits that these functions are conveyed through cortico-basal ganglia thalamocortical loops (Alexander and Crutcher, 1990). Neural firing patterns in basal ganglia output circuits during motor tasks often show complex associations with behavior, including rate changes linked to sensory events as well as with movement preparation and execution (Jaeger et al., 1993, 1995; Schmidt et al., 2013; Mirzaei et al., 2017). Further, cortico-basal ganglia circuits have been found to control interval timing through ramping activity leading up to expected events (Emmons et al., 2017).

The motor thalamus provides a critical link between the BG and the motor and premotor cortices (Theyel et al., 2010; BoschBouju et al., 2013; Guo et al., 2017). In rodents, the areas of motor thalamus receiving direct input from basal ganglia, consist 
of the ventromedial (VM) and rostro-ventral portion of the ventral anterior (VA)/ventral lateral (VL) nuclei [i.e., ventro-anterior-lateral (VAL); Kuramoto et al., 2011]. We abbreviate this area as $\mathrm{VM} / \mathrm{VAL}$ in the following. In rodents, VM/VAL is entirely made up of glutamatergic neurons projecting to cortex (Bosch-Bouju et al., 2013) and receives an inhibitory projection from the substantia nigra reticulata $(\mathrm{SNr})$ and entopeduncular nucleus, the two main output nuclei of the BG (Sakai et al., 1998; Kuramoto et al., 2011; Guo et al., 2018). These GABAergic BG output neurons have high tonic firing rates in vivo (Delong, 1971; Ruskin et al., 2002; Lobb and Jaeger, 2015), resulting in tonic thalamic inhibition through powerful $\mathrm{GABA}_{\mathrm{A}}$ synapses (Bodor et al., 2008).

Recent studies in mice show that the VM thalamic nucleus forms a closed loop with anterolateral premotor cortex (ALM) and that this loop is required for persistent activation of neural activity related to movement preparation and decision-making during the delay epoch of correct trials in a cued left/right lick task (Guo et al., 2017). However, the processes by which the basal ganglia interact with this cortico-thalamic motor-planning loop may influence specific aspects of this motor task, such as the errors related to the proper timing of lick action release, remain unknown.

To better understand the specific role of the nigro-thalamocortical pathway in motor control, we recorded thalamic singleunit activity with 32-channel silicon probes and optogenetically stimulated nigral terminals in VM/VAL during the delay epoch of a cue-guided decision-making right/left lick task in mice, similar to the task used in the study by Guo et al. (2017) to explore ALM-VM interactions. We confirm that VM/VAL spiking showed a prominent ramping of activity during the delay epoch in anticipation of the Go cue. Importantly, we found that this activity was weaker in trials where the mice failed to initiate a lick [omission (omi) trials], and stronger in trials where licking was premature [impulsive (imp) licks]. Stimulating SNr terminals in $\mathrm{VM} / \mathrm{VAL}$ during the delay before the Go-cue lead to a decrease in impulsive licks as well as an increase in the number of omissions. Analysis of single-unit activity revealed that the optogenetic (opto) stimulation produced spike rate changes in VM thalamus that were similar to the signature of spontaneously occurring omission trials. Overall, our results indicate that premotor ramping activity in the VM/VAL thalamus contributes to controlling the proper timing of action release and that this process is likely modulated by basal ganglia input.

\section{Materials and Methods}

Mice. All mice used were male and female 3- to 6-month-old transgenic Vgat-ires-Cre knock-in mice (Vong et al., 2011) expressing Crerecombinase under a vesicular GABA transporter (VGAT) promoter specific to GABAergic neurons (stock \#028862, The Jackson Laboratory). Animals were maintained on a C57BL/6 background and kept on a $12 \mathrm{~h}$ reverse light/dark cycle. During the behavioral training periods, animals were water restricted to $85 \%$ of their body weight. Food was available ad libitum.

Stereotaxic surgeries. The analgesic Buprenorphine SR-LAB (1 mg/ $\mathrm{kg}$, s.c.) was administered preoperatively. Mice were deeply anesthetized with isoflurane. Mice were placed on stereotaxic apparatus (Kopf) on a heat pad. Ophthalmic ointment was applied to the eyes. The level of anesthesia, temperature, and breathing were monitored. A scalp incision revealed the skull, which was cleaned and dried. A pinhole craniotomy was drilled above the $\mathrm{SNr}$ [anteroposterior (AP), $-3.4 \pm 0.5 \mathrm{~mm}$; mediolateral (ML), $-1.4 \pm 0.5 \mathrm{~mm}$, relative to bregma]. A viral vector containing the transgene (rAVV2-EF1a-DIO-hChR2(E123T/T159C)-EYFP) was injected $(200 \mathrm{nl})$ into the left $\mathrm{SNr}(\mathrm{AP},-3.40 \pm 0.25 \mathrm{~mm}$; ML, $-1.40 \pm 0.25 \mathrm{~mm}$; dorsoventral, $-4.40 \pm 0.10 \mathrm{~mm}$ relative to bregma).
After the injection, a custom-machined aluminum head bar was cemented onto the skull using dental adhesive resin (Metabond C\&B, Parkell Products). A pinhole craniotomy was made above the cerebellum area for the ground wire (tungsten). The head bar was secured with more dental cement, and the entire skull area was protected with a thin layer of cement. Antibiotic ointment was applied around the cemented area. An additional craniotomy above the VM/VAL thalamic area (AP, $-1.25 \pm$ $0.50 \mathrm{~mm} ; \mathrm{ML},-0.75 \pm 0.50 \mathrm{~mm}$ relative to bregma) was performed a few days before acute electrophysiological data acquisition, and protected with Kwik-Cast silicon sealant (WPI) outside of recording sessions.

Behavioral training. The task was computer controlled using a custom-made LabView (National Instruments) interface. Mice were handled daily in the experimental room and progressively habituated to the head-fixation procedure in a transparent plastic tube (pretraining procedure adapted from the study by Guo et al., 2014a). The training procedure was then continued daily for 6-8 weeks and divided into three segments. (1) Mice learned to respond to the Go-cue sound by licking, within $1.5 \mathrm{~s}$ after the cue onset, on a single centered lick spout to obtain a water droplet. (2) Mice learned the association between a mild air puff (oriented pseudorandomly at each trial toward either the left or the right whiskers) and the reward side (two lick spouts located at $2.5 \mathrm{~mm}$ left and right of the snout and $5 \mathrm{~mm}$ below the snout). If the trial started by a left air puff, a first lick to the left was rewarded. An algorithm automatically changed the probability of left and right trials to balance the error probability on both sides. In this algorithm, the moving average of correct performance was computed and trials to the side where the mouse performed less reliably were increased in frequency. (3) Mice learned to withhold their licking during a delay epoch (increasing by steps of 250 $\mathrm{ms}$ until a delay of $750 \mathrm{~ms}$ was reached).

The transition from one training segment to the next occurred when a criterion of $70 \%$ was reached. In each segment, errors (licking to early or wrong side) were signaled by an error sound and followed by a time penalty $(6 \mathrm{~s})$. All training and recording sessions were performed in a dark behavioral box isolated from light, sounds, and electrical noise.

Data acquisition. All raw signals from electrodes and task-related data [events signaled by transistor-transistor logic (TTL) pulses] were acquired at $20 \mathrm{kHz}$ through an RHD2000 Recording System (Intan Technologies). Electrophysiological data were notch filtered at $60 \mathrm{~Hz}$. A lick sensor triggered a TTL pulse when the tongue contacted the left or right lick spout. The measurement used a custom-made electronic circuit measuring a change in resistance between the mouse and the metal lick tube. Trial start events were recorded on the Intan RHD2000 Recording System as TTL pulses to allow precise trial alignment during analysis. The same TTL pulse also triggered the video acquisition for each trial. Air-puff and Go-cue events issued by the LabVIEW program were recorded through TTL pulses on the RHD2000 Recording System board as well to allow precise event alignment with spiking activity.

Video data (Movies 1, 2, 3, 4) of the mouse licking and other orofacial behaviors were acquired at $25 \mathrm{~Hz}$ (ace1300 camera, Basler), under infrared LED illumination. A recording of 100 frames was triggered at each trial start (TTL rising edge). We used Python-based DeepLabCut (Nath et al., 2019) in a following offline analysis to extract snout, tongue, and whisker movements from videos (Movie 1).

Electrophysiological signals were obtained by acute insertion of silicon probes (optrodes) made of four shanks separated by $200 \mu \mathrm{m}$ with eight channels per shank separated by $100 \mu \mathrm{m}$ (A4x8-7 mm-100-200177, NeuroNexus). A $200-\mu \mathrm{m}$-diameter optic fiber [0.22 numerical aperture (NA)] was mounted $200 \mu \mathrm{m}$ above the first channel on the third shank. An internal reference channel was located $200 \mu \mathrm{m}$ above the first channel on the second shank. Neuronal activity was recorded from the $\mathrm{VM} / \mathrm{VAL}$ thalamus. Recordings were started $20 \mathrm{~min}$ after probe insertion to allow the electrode-brain interface to stabilize. Skull screws were cemented above the cerebellum and used as ground and external reference in case the internal reference would fail.

Spike sorting. Each spike exceeding the detection threshold (4 SDs away from the mean raw signal) was represented by 40 points centered on the minimum value. Single-unit spikes were extracted and sorted in MATLAB (MathWorks) using WaveClust, a method for spike detection [pass band filter; minimum frequency $(\mathrm{fmin})=600$, maximum frequency 
$(f \max )=8000$, minimum $\mathrm{SD}=4$, maximum $\mathrm{SD}=30$, filter order $=4$ ] and sorting ( $\mathrm{fmin}=300, \mathrm{fmax}=4000$, filter order $=2$ ) with wavelets and superparamagnetic clustering (Quiroga et al., 2004; Quiroga, 2012). After the automatized clustering procedure, each cluster was manually reinspected to eliminate poorly defined or noisy clusters. Following the study by Sukiban et al. (2019), the spike-sorting quality metric used was the percentage of spikes violating a $3 \mathrm{~ms}$ refractory period (i.e., spikes occurring $<3 \mathrm{~ms}$ following the previous spike). The vast majority of units showed $<1 \%$ refractory period violations (see Fig. $2 C, D$ ).

Optogenetic stimulation. For all photostimulation experiments, we used a blue laser ( $473 \mathrm{~nm} ; 10 \pm 2 \mathrm{~mW}$ at fiber end; Dream Laser). An optical fiber patch cord (diameter, $200 \mu \mathrm{m}$; $0.22 \mathrm{NA}$; Doric Lenses) was directly attached to the optrode with a ceramic sleeve. Aluminum foil was carefully wrapped around the sleeve to further prevent light from spreading. In-task optogenetic stimulation (see Fig. 7) consisted of 1-slong continuous pulses delivered randomly in $20 \%$ of the trials and starting $500 \mathrm{~ms}$ before the onset of the Go cue.

To confirm localization of the optrode in the area responsive to nigral optogenetic stimulation, a train of $\sim 50$ laser stimulations ( $1.5 \mathrm{~s}$ laser ON, 1.5 s laser OFF) was given outside of the task and examined online for changes in multiunit spiking. These stimuli were later also used post hoc to analyze 89 isolated single units to determine spike rate changes for laser ON versus laser OFF periods (statistical significance was determined with a Wilcoxon test).

Histology. Mice were killed by an overdose of ketamine/xylazine mix (administered intraperitoneally), then perfused intracardially with $4 \%$ paraformaldehyde (PFA) in PBS for tissue fixation. The brain was extracted and stored overnight in 4\% PFA and then transferred to a $30 \%$ sucrose solution for storage at $4^{\circ} \mathrm{C}$. Brains were sliced $(40 \mu \mathrm{m})$ using a microtome and stained by two different methods: Nissl (cresyl violet) and immunohistologically using anti-GFP (peroxidase). We used confocal imaging to observe GFP fluorescence on unstained slices. Electrode tracks were reconstructed post hoc based on histologic results. The silicon probe shanks had a $50 \mu \mathrm{m}$ thickness, which makes them relatively stiff and unlikely to bend compared with commonly used $15-\mu \mathrm{m}$-wide silicon probes. The depth of insertion was controlled through a Sutter MP 285 micromanipulator with a micrometer-precise electronic readout during the experiment and confirmed by histologic track reconstruction. After histologic reconstruction, we used the Allen Brain Atlas to register the position of each channel as described by Shamash et al. (2018) using landmarks such as the midline, the mammillothalamic tract $(\mathrm{mt})$ fiber bundle, and the hippocampus.

Experimental design and statistical analyses. Numerical values obtained by averaging are denoted as follows: mean \pm SD. Statistical tests were conducted with an $\alpha$ of 5\%, two sample and two tailed, except when specified otherwise. Statistical test results are described as significant in the text where $p<0.05(*<0.05, * *<0.005$, $* * *<0.0005)$. We used a $t$ test for parametric tests and the Wilcoxon rank-sum test for nonparametric tests. A Bonferroni correction was applied when multiple comparisons were performed. Shaded areas in plots indicate 1 SEM, except when specified otherwise. Specifics on the statistical methodologies and software used for various analyses are described in the corresponding sections in Results, Materials and Methods, and figure legends.

Data come from a total of five mice in this study. Our study was not designed to compare across sexes, so we randomly picked one male and four female mice.

Guo et al. (2017) have shown that VM thalamic nuclei are functionally interconnected with ALM cortex during a left/right lick task with a delay. We decided to use the same task design to build on prior results and study the effect of nigral input on the VM activity. Note that in this study we decided not to analyze the error side (eSi) trials that had very low numbers. We also decided not to separate trials into left and right choice because this analysis has been already done by Guo et al. (2017). Instead, here we decided to focus our attention on the error trials related to timing, impulsivity, and omissions. Further dividing these error trials into left and right trials would result in in numbers too low for statistical analysis. Our dataset contains 15 recording sessions (five mice). Among them, 11 sessions were recorded with optogenetic stimulation (four mice, all female). Because optogenetic stimulation further decreased the already low probability of impulsive trials, in some specific analyses requiring impulsive trials during optogenetic stimulation we had to select five sessions (three mice) with at least three trials of each type (see Fig. 7E).

All subsequent data analysis was performed using custom MATLAB (MathWorks) scripts. Raster plots and perievent time histograms (PSTHs) were centered on the onset of task events [either the Go cue or the decision lick (the first lick detected by the lick sensor)]. A spike rate function (SRF) was obtained by converting the values from the $1 \mathrm{~ms}$ binned PSTHs to a spike rate function in spikes per second (Hertz) and smoothing the instantaneous rates with a $75 \mathrm{~ms}$ Gaussian kernel, except see Figures $4 B, 5 B$, and $7 D$, where we used a $40 \mathrm{~ms}$ Gaussian kernel to match the $40 \mathrm{~ms}$ video frame acquisition time. For each neuron, we computed an average SRF across all trials (SRFalltr) and the corresponding averaged SD (SDalltr), as well as a value of baseline (frBaseline) obtained by averaging the firing rate during the intertrial interval (ITI) period $(2 \mathrm{~s}$ before each trial start without any licks) across trials.

The $z$ scores were obtained by normalizing the mean firing rate to the mean baseline value, as follows:

$$
\text { Zscore } 1=\frac{\text { SRFalltr1 }- \text { frBaseline }}{\frac{\text { SDalltr } 1}{\sqrt{\text { ntrial } 1}}}
$$

and $\mathrm{z}$ score differences $(\mathrm{dZ})$ values were obtained by subtracting $z$ scores obtained from different trial types [e.g., $\mathrm{dZ}=z$ score for omission trials (Zomi) $-z$ score for correct trials (Zcor)], as follows:

$$
\mathrm{dZ}=\text { Zscore } 1-Z \text { score } 2 .
$$

To assess the information contained in single-trial spike trains regarding subsequent trial outcomes (see Fig. 6), we trained a binary, linear classification model using a logistic learner (fitclinear MATLAB function). We computed the accuracy of the classification for different sizes of cell ensembles (from 1 to 20 cells recorded simultaneously) by running 100 iterations with a 10 -fold cross-validation for each group size. We ran the classifier for each session individually and then averaged them across sessions. At each iteration, a random subgroup of cells was selected from the available population of cells recorded simultaneously in that session. A shuffle control was obtained by assigning spike trains to a random trial outcome. The learning process was repeated for the shuffled data using the same procedure. For this analysis, we used 12 sessions (five mice) with at least $20 \mathrm{VM} / \mathrm{VAL}$ cells recorded simultaneously.

Data availability. Custom MATLAB scripts and data sets used in this study can be accessed on reasonable request addressed to the corresponding authors.

\section{Results}

\section{Omissions and impulsive licking are naturally produced by head-fixed mice engaged in a delayed lick decision task}

To study the role of the nigro-thalamic pathway in motor control, we used a licking task in which mice had to make a binary choice based on sensorial discrimination and with a delay separating perception and action. Similar tasks have been used and validated by previous studies in head-fixed mice (Guo et al., 2014a,b, 2017; Li et al., 2015).

In our version of the task (Fig. 1A, Movie 1, Movie 2, Movie 3, Movie 4), the mice received a mild air puff (duration, $750 \mathrm{~ms}$ ) on left or right whiskers and needed to withhold their licking during the air puff and the following delay epoch (duration, $750 \mathrm{~ms}$ ). A sound Go cue indicated the end of the delay and the start of the licking epoch (duration, $1500 \mathrm{~ms}$ ). During the licking epoch, the mouse had to indicate that they remembered the side of the previous air-puff stimulation by first licking into the lick port located on the same side (decision lick). A water reward was 
A

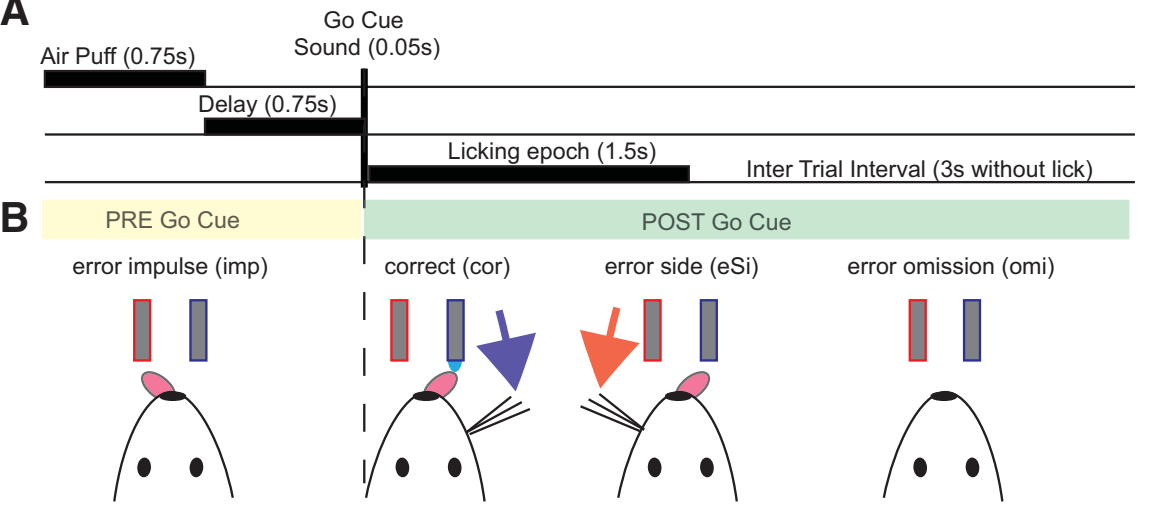

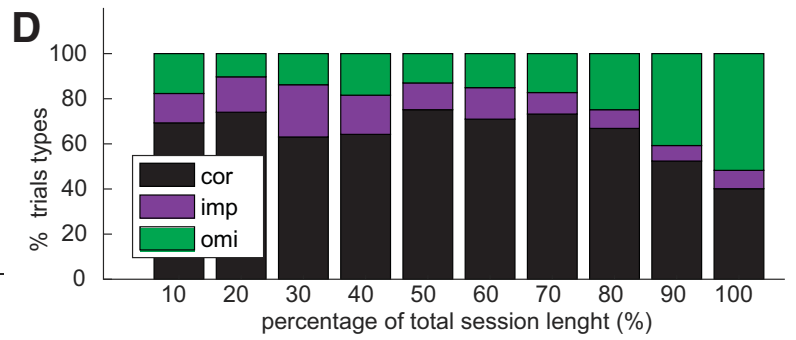

$E$

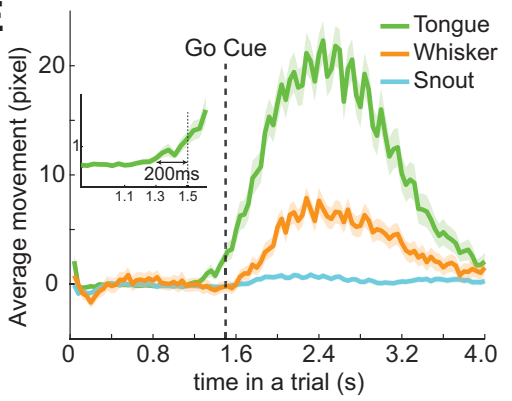

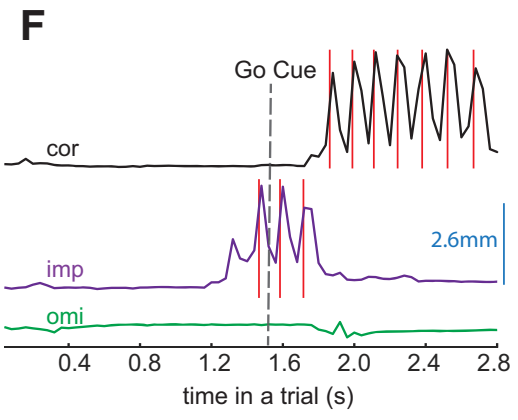

Figure 1. Mouse behavior in tactile cued choice delayed the response task. A, Schematic time course of a task trial. At the start of each trial, a mild puff of air (air-puff epoch) was delivered pseudorandomly on the left or on the right whiskers of the head-fixed mouse for $75 \mathrm{~s}$. Once the air puff stopped, the mouse had to continue to withhold licking during the delay epoch $(750 \mathrm{~ms})$. The end of the delay was indicated by a Go signal (sound) after which the mouse could initiate the decision lick denoting the choice of side (licking epoch). If the decision lick was for the correct side spout during the licking epoch, a water reward was delivered. Trials were separated by a $3 \mathrm{~s}$ minimum ITI. Any licks during the ITI led to a restart of the counter and to starting the next trial at $2 \mathrm{~s}$, ensuring that at the onset of each trial the mice had completely stopped licking from the previous trial. B, Schematic of the four possible trial outcomes: (1) error impulsive lick (before Go cue); (2) correct lick; (3) error lick on the wrong side; and (4) error because of no licking during the licking epoch (omission). C, Bar graph (mean and SEM) of the behavioral results ( $n=15$ sessions, five mice, 2060 trials) obtained after training periods, during electrophysiological recording. Top, Percentage over the total amount of correct and eSi trials. We found significantly more correct trials (cor $=87.4 \pm 10.4 \%$; mean \pm SD) Two-sample $t$ test $(n=15 ; * * * p<0.0001)$. Bottom, Percentage among all trials, Errors because of choice of side (eSi) were less frequent than errors related to timing (imp + omi). $\boldsymbol{D}$, Distribution of trial outcomes across sessions. Averaged across 15 sessions, five mice. eSi trials were not included. $\boldsymbol{E}$, Average DeepLabCut tracks from the video analysis of tongue, snout, and whisker movements during correct trials (six sessions from four mice). Data are shown as absolute values of changes in tracked position in pixels relative to baseline along the $y$-axis (top-to-bottom movement in Movies 1, 2, 3, 4) for tongue and snout movement and along the $x$-axis (right-to-left movement in Movies 1, 2, 3, 4) for whisker motion. For each trial, 100 frames were taken at $25 \mathrm{~Hz}$. The inset shows that tongue movement can be detected up to $200 \mathrm{~ms}$ before the $\mathrm{Go}$ cue in some trials. $\boldsymbol{F}$, Examples of DeepLabCut tongue tracking (raw data) for cor, imp, and omi single trials. Note that a typical correct lick sequence starts by one decision lick followed by a bout of several retrieval licks (black trace). The lick sensor TTL activation is represented by vertical red lines. Scale bar (vertical blue), $2.6 \mathrm{~mm}$ (the distance between the spout and the mouth). Note that when the lick did not reach or miss the spout, the lick sensor did not activate, as shown by the first tongue movement in the impulsive trial. Note that our $25 \mathrm{~Hz}$ (40 ms/frame) video acquisition was not fast enough to determine the exact onset of tongue protrusion with respect to lick sensor detection, but that the approximate timing of the tongue trajectory is fully consistent with the timing of tongue protrusion starting $33 \mathrm{~ms}$ before spout contact for cue-evoked licks seen with videos taken at $1 \mathrm{kHz}$ (Bollu et al., 2019).

obtained, only if the decision lick was to the correct side and not later than $1.5 \mathrm{~s}$ after the Go cue. An ITI of a minimum of 3 $\mathrm{s}$ was given between trials, and the next trial started only after the mice stopped licking for $2 \mathrm{~s}$ (Fig. 1A). The mice could succeed (cor trials) or commit the following three types of errors (Fig. 1B): lick too early (imp trials), lick too late or no lick (omi trials), or lick to the wrong side (eSi). Mice were trained for 6-8 weeks for an average of $25 \pm 3$ sessions, until they reached a $70 \%$ criterion performance for licking to the correct side. After training, five mice were successfully recorded during the task across 2060 trials in 15 sessions (137 \pm 40 trials per session). In trials that included a decision lick in the licking epoch, mice were licking the correct side first in $87 \%$ of trials, while making $13 \pm 10 \%$ of side errors (Fig. 1C). Importantly, omission and impulsive trials did not vanish after training (Fig. 1C).

Omission and impulsive trials were present throughout the entire behavioral sessions. However, some shift in failure types did occur between the beginning and end of sessions (Fig. 1D). Specifically, the occurrence of omission trials increased toward the end of sessions, whereas impulsive trials were slightly more frequent earlier. These effects are likely because of decreasing thirst and, hence, the urgency to perform during each session as more and more water was consumed. Across all sessions, we found that the proportion of errors related to timing of action (omi + imp; $43.2 \pm$ $14.7 \%$ ) was significantly higher than the proportion of errors because of touching the lick spout on the wrong side first (eSi, $8.9 \pm 8.1 \%)$, two-sample $t$ test $(n=15$; $* * * p<0.00005$; Fig. 1C). The nonvanishing proportion of these impulsive and omissive trials gave us the opportunity to study VM/VAL single-unit spiking responses during these two types of error trials, both related to the timing of action release.

Video recordings $(25 \mathrm{~Hz})$ were used to complement the information provided by our lick sensor and detect potential prelick movements of the snout, whiskers, and tongue itself. The trial average of movement traces for correctly performed trials obtained with DeepLabCut tracking (Movie 1) revealed the infrequent but possible presence of tongue movement up to $200 \mathrm{~ms}$ before the Go cue, but did not reveal snout or whisker preparatory movement (Fig. 1E, Movie 1, Movie 2, Movie $3)$. We also compared the lick sensor readings to the tongue movement (Fig. $1 F)$ and found that DeepLabCut 


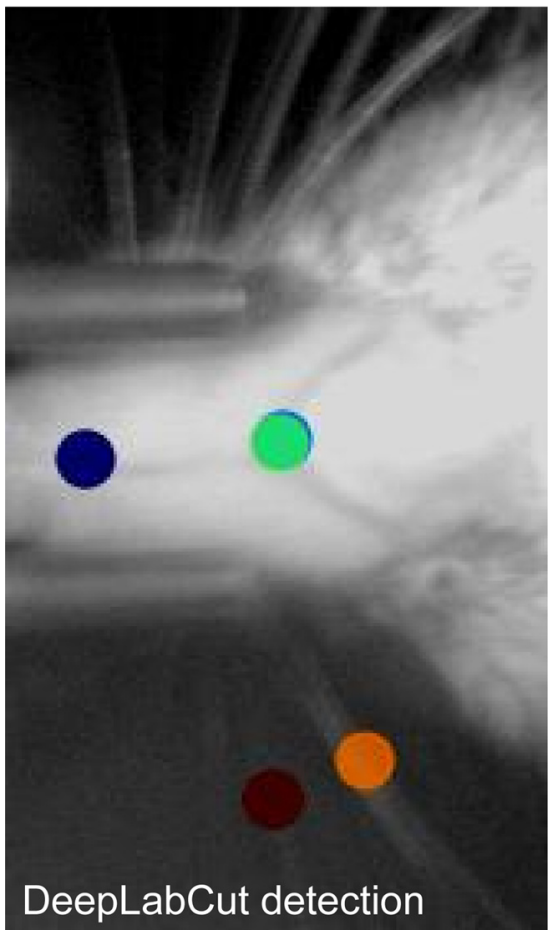

Movie 1. Example video with deeplabcut labels, tracking the snout (dark blue), mouth (light blue), tongue (green), and whiskers (orange and red). View from bottom of the mouse head during the task. The video is display at real speed ( 25 frame per second). [View online]

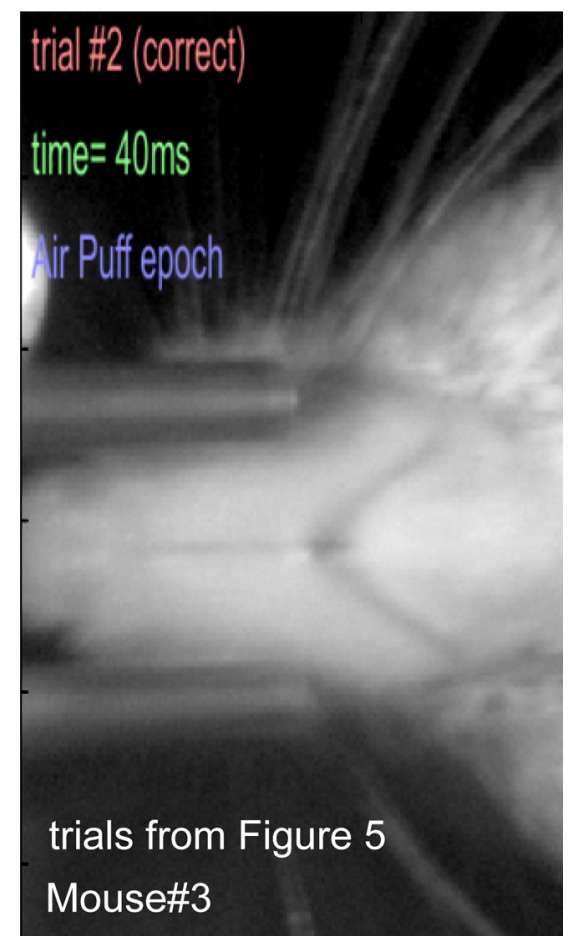

Movie 2. Video corresponding to trials shown in Figure $5 A$ from mouse \#3. View from bottom of the mouse head during the task. Colored dynamic text display (top left corner) indicates the trial number and trial type (red), the time from trial start (in green), the type of task event currently occurring (violet). The video is display at slower speed (10 frame per second). [View online]

tracking revealed predecision partial tongue protrusion movement in $46 \%$ of 450 analyzed correct trials from four mice. This tongue movement started on average $160 \pm 60$ ms before the TTL lick sensor touch detection. Only in $7 \%$

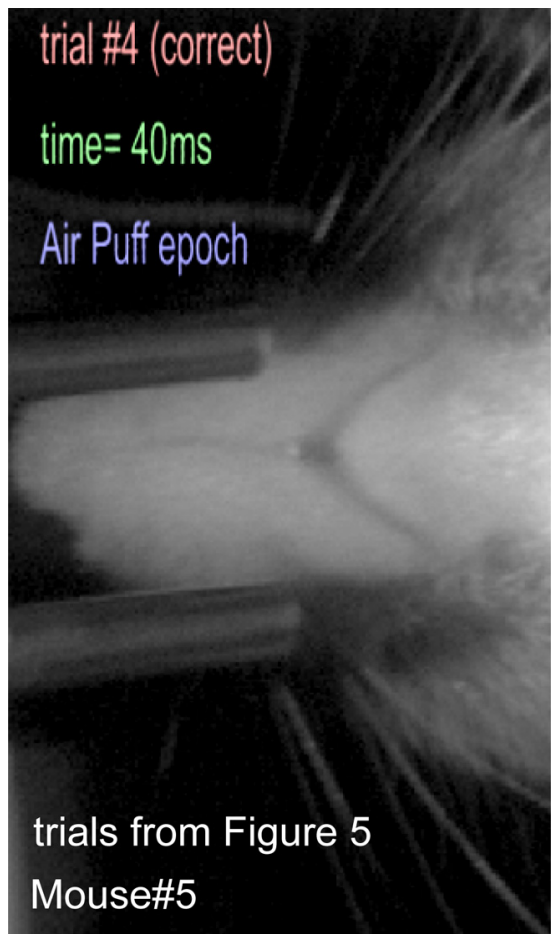

Movie 3. Video corresponding to trials shown in Figure $5 B$ from mouse \#5. Legend similar to Movie 2. [View online]

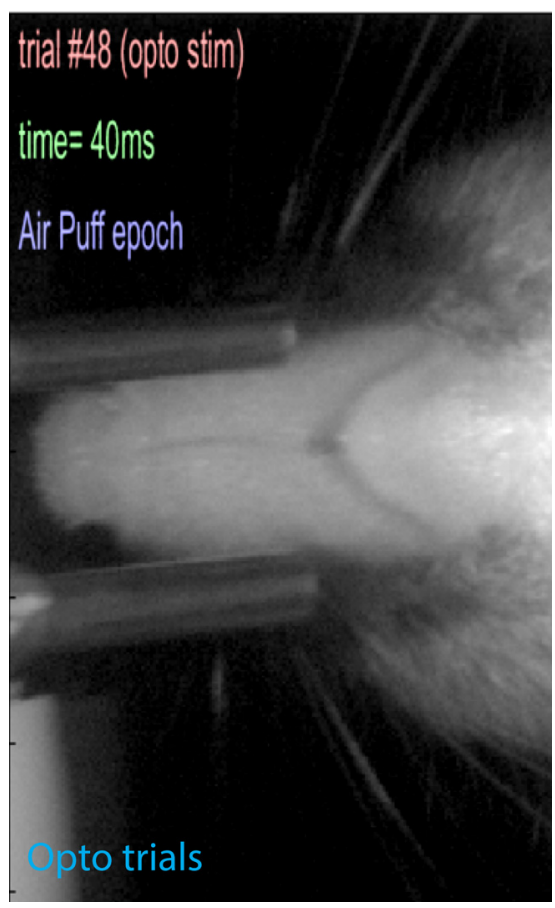

Movie 4. Video of examples trials with optogenetic stimulation from mouse \#5 to complement results from Figure 7. The Laser stimulation are indicated by the apparition of a light blue text (middle left). Legend similar to Movie 2. [View online]

of the trials a lick was observed before the Go cue (at 68 $\pm 34 \mathrm{~ms})$. These results indicate that preparatory movements (including partial licks) were generally confined to a $200 \mathrm{~ms}$ period before the decision lick detected by our electronic lick sensor (Fig. 1E,F). 
A

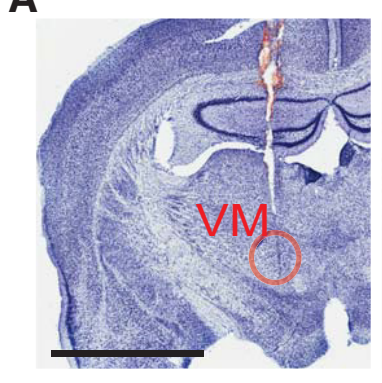

B

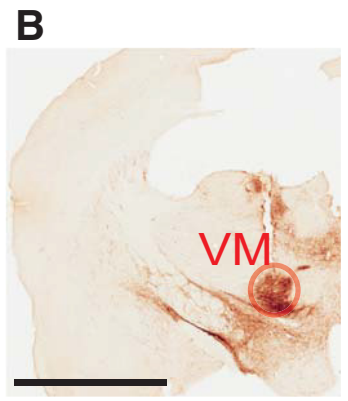

C

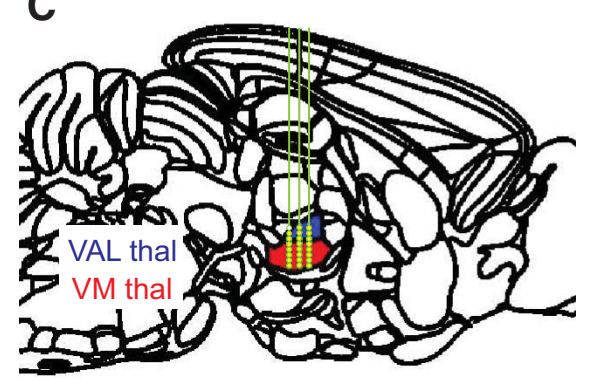

$\operatorname{rpv}=0.1 \%$

E
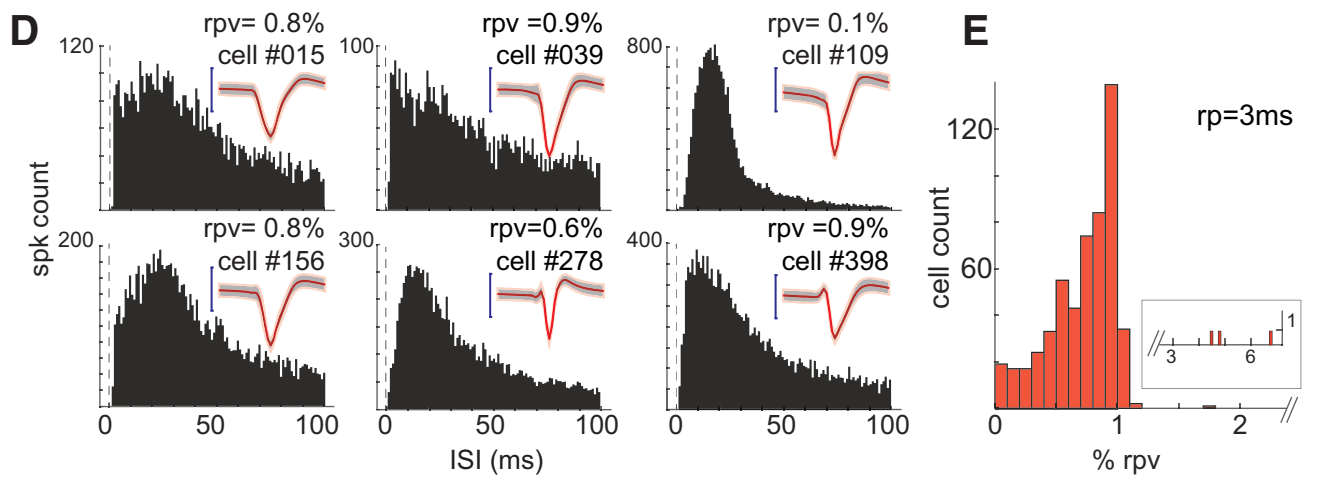

Figure 2. Histologic reconstruction and single-unit quality metrics. A, Nissl stain from a representative coronal brain slice revealing the position of an electrode track within the VM thalamus (red circle). Scale bar, $2 \mathrm{~mm}$. B, Immunohistochemical image from a representative coronal brain slice using anti-GFP antibody revealed GFP expression because of AAV vector injection into SNr (see Materials and Methods) with horseradish peroxidase (darker area). The location of GFP confirms the presence of GABAergic SNr terminals in VM thalamus (red circle) where the electrode track is located. C, Example of electrode track reconstruction, and registration to the Allen Brain Atlas. Sagittal schematic representation of a mouse brain with each brain area delimited by black lines. The VM and VAL thalamus (colored red and blue, respectively) contained three electrode shanks (green lines) and their channels (green circles). Channels were spaced by $100 \mu \mathrm{m}$. Shanks were spaced by $200 \mu \mathrm{m}$. D, ISI distribution for six VM/VAL single units. For each unit, a \% rpv of $3 \mathrm{~ms}$ is indicated. The average spike wave is also depicted (red traces). Each spike trace is $2 \mathrm{~ms}$. Calibration: 100 $\mu \mathrm{V}$. E, Distribution of $\% \mathrm{rpv}$ for all $462 \mathrm{VM} / \mathrm{VAL}$ thalamic neurons recorded. The inset shows only 3 units with $>2.5 \% \mathrm{rpv}$, which should not be considered as an isolated single unit.

Dynamic changes in thalamic neuronal activity during correct trials of the task include a delay-specific ramping in firing rate preceding action release

To determine the relation of VM/VAL activity with our delayed response task, we recorded the activity of $462 \mathrm{VM} / \mathrm{VAL}$ thalamic neurons during the task. Extracellular recordings were performed using four shank silicon probes (Fig. 2). After the completion of recording sessions, electrode tracks were revealed through histologic sectioning $(40 \mu \mathrm{m})$ and both Nissl staining (Fig. $2 A)$ and immunohistochemistry (Fig. $2 B$ ) for all mice. Once electrode placement was assessed by locating tracks with respect to anatomic landmarks, we used the Allen Brain Atlas to register the results with stereotactic coordinates (Fig. 2C). Only cells registered with VM or VAL coordinates by the Allen Brain Atlas were used for further analysis. We then plotted the interspike interval (ISI) distribution (Fig. 2D) for VM/VAL units isolated with WaveClust (see Materials and Methods) and quantified their spike-sorting quality by computing the percentage of spikes with a $3 \mathrm{~ms}$ refractory period violation (percentage rpv; Fig. $2 D, E$ ). We found that with the exception of 5 of 462 units our unit separation showed $<1 \% \mathrm{rpv}$ (Fig. $2 E$ ), which is less than the $2.5 \%$ deemed acceptable to represent isolated single units (Sukiban et al., 2019).

First, we analyzed spike rates for the correct trials of the task (Fig. 3). We found that $>80 \%$ of VM/VAL neurons responded by significantly increasing their spike rate relative to baseline ( $z$ score, $>3$ ) during the time course of a correct trial. The remaining neurons presented either no significant change during the task or presented a significant decrease of activity (Fig. 3A). Fifty-four neurons presented a complex dynamic of changes of activity including both a significant increase and a decrease in spike rate (Fig. $3 A$, intersection of the Venn diagram).
We categorized neural responses during the correct trials of the task. Consistent with previous results (Guo et al., 2018), we found a great diversity of responses at the single-unit level (Fig. $3 B$ ) including a clear delay-specific ramping pattern of activity preceding movement. We categorized three main response types (type I, air puff; type II, delay; type III, licking), and we found that a large majority of neurons showed a significantly increased spike rate during multiple task epochs (Fig. 3C). Type II neurons with significantly increased spike rates during the delay in most cases also showed increased spike rates during other task epochs, notably a peak spike rate in the following licking epoch (Fig. 3B, cell \#015).

We plotted the average $z$ scored spike rate function over all neurons recorded during the task, which revealed a peak during the air puff stimulation epoch in addition to a clear ramp up during the subsequent delay epoch with a peak in the following licking epoch (Fig. 3D, black curve). After eliminating units with significant decreases or no change in spike rates during the task from the average, these peaks remained similar in shape and became more pronounced (Fig. $3 D$, red curve). The average inhibitory responses were also associated with sensory cue and licking epoch onsets, but no inhibitory ramping during the delay was observed (Fig. 3C, green curve). We decided to focus our further analysis on the 338 neurons that exclusively showed excitation in relation to behavior (Fig. $3 A$, red group, $D$, red curve).

By sorting the activity by first occurrence of a $z$ scored threshold during the delay we observed that most of the 338 neurons ramped up in spike rate before the decision lick, indicating that they were related to preparatory activity preceding the action release (Fig. $3 E$ ). In addition to ramping, VM/VAL also tended to overrepresent the start of the trial corresponding to the onset of the air-puff epoch as shown by the distribution of peak activity rate (Fig. $3 F$ ), which 
A
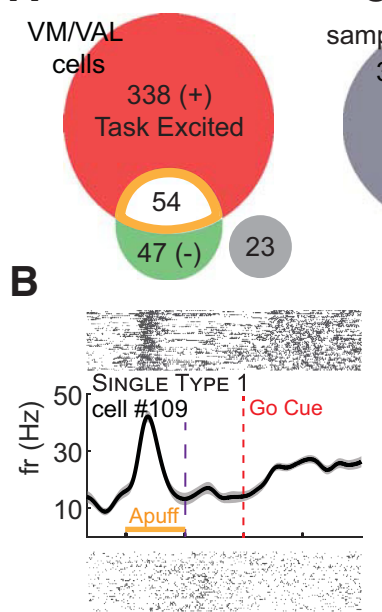

SINGLE TYPE 2

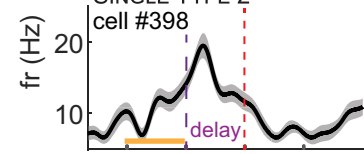

SINGLE TYPE 3

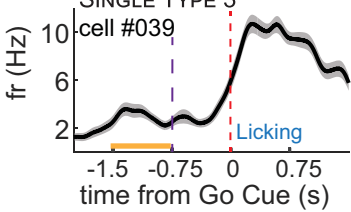

C
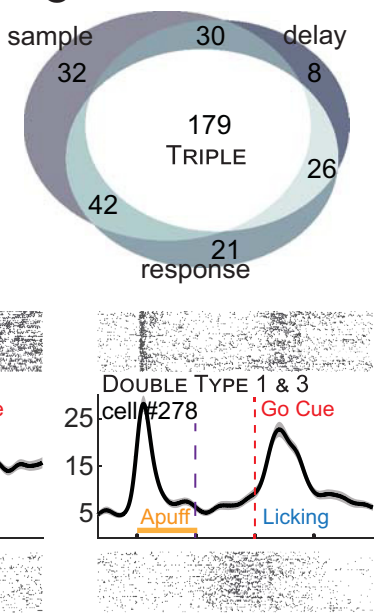

DOUBLE TYPE 2 \& 3 25 cell \#156

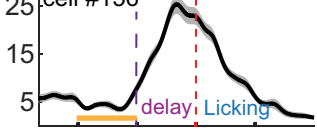
TRIPLE TYPE $122 \& 3$

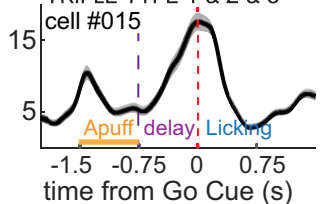

D

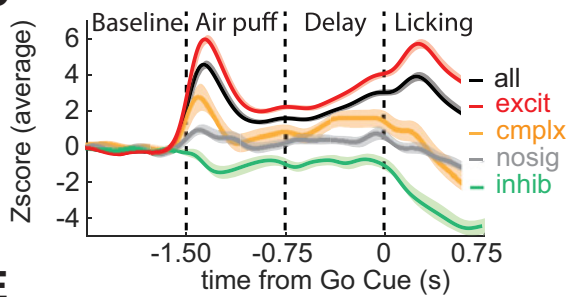

E

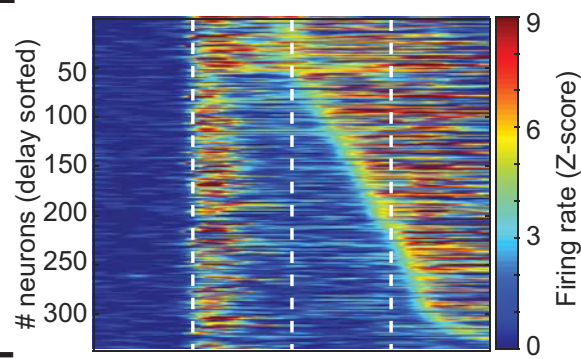

F

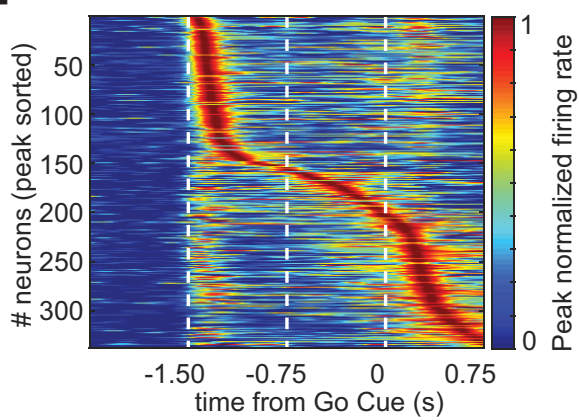

Figure 3. VM/VAL thalamic neuronal responses during correct trials reveal the ramping pattern during the delay of the task at both single-unit and population levels. $A$, Venn diagram representing different types of single-unit firing rate modulation during the task. Red, Task-excited cells (firing increase only); green, task inhibited (firing rate decreased only); orange, cells with complex task modulation (both increases and decreases firing rate); and gray, cells not significantly modulated (462 VM/VAL neurons from 15 sessions, five mice). $\boldsymbol{B}$, Raster plots and spike rate functions for six VM/VAL single units, providing representative examples of the six types of dynamic neuronal responses observed during the correct trials of the task. The orange horizontal bar represents the time of air-puff delivery, violet and red dashed lines represent the onset of the delay and Go cue, respectively. ISI distribution and \% rpv of these units are given Figure $2 D$. C, Venn diagram representing the different dynamic response types (of the 338 VM/VAL neurons excited during the task; red in $\boldsymbol{A}$ ). We found units with significant modulation ( $z>3$ ) only during a single epoch: air puff (type 1), delay (type 2), or licking (type 3), or single units showing significant modulation in multiple epochs (double or triple response types). $\boldsymbol{D}$, Average firing rate normalized to baseline ( $\boldsymbol{z}$ score) as a function of time for neuronal groups defined in $\boldsymbol{A}$ based on their firing rate properties. $\boldsymbol{E}$, Trial-averaged heat map of baseline normalized firing rates for all units sorted by the first occurrence of $z>3$ during the delay. This map shows the broad distribution of ramping activity initiation between different units. $\boldsymbol{F}$, Trial-averaged heat map of the peak (maximum) normalized firing rate for all units sorted by peak time. This map shows that a large proportion of units had their spike rate maximum shortly after the onset of the air puff or during the licking epoch. Note that this timing of maximal firing rates does not preclude ramping activity during the delay epoch or the presence of additional distinct (but smaller) peaks in spike rate.

could be related to sensory signal processing and/or to a potential trial time estimation mechanism (see Discussion).

Together, these results indicate that VM/VAL spike rates showed a prominent ramping of population activity during the delay of correct trials, which preceded the early partial licks observed in a subset of trials (Fig. 1E,F).

\section{VM/VAL delay-specific ramping activity best relates to the} timing of the decision lick and not to the go-cue signal onset Ramping activity during the delay could reflect an anticipation of the Go-cue signal or the timing of the decision lick. To disambiguate between these two possibilities, we centered the spike raster and spike rate plots on the decision lick (Fig. $4 A, B$ ). We found that the ramping showed its peak shortly before the decision lick even in imp trials where the lick preceded the Go cue (Fig. 4A). The ramping of the population average for correct trials started $(z$ score, >1; Fig. 4A) at $648 \mathrm{~ms}$ before the lick onset and had its peak $160 \mathrm{~ms}$ ( $n=60$ neurons) before the lick onset (lick onset defined by the lick sensor touch; Fig. 1F). These results were obtained during correct trials both at the population level $(n=60$ neurons; Fig. $4 A$, black curve) and at the single-unit level (Fig. $4 B$ ). Importantly, we obtained a similar time course in both impulsive and correct trials (Fig. $4 A$ ), indicating that the ramping activity anticipated the release of the lick action and not the Go-cue signal.

\section{VM/VAL delay-specific ramping activity is altered in impulsive and omission trials}

To further confirm the finding that ramping relates specifically to the timing of motor action release in the context of our licking task, we wondered whether the ramping found in correct trials occurred early in impulsive trials and was reduced or absent in omission trials. To address this question, we analyzed changes in spike rates during the delay of error trials and compared the results to correct trials for $234 \mathrm{VM} / \mathrm{VAL}$ neurons (Fig. 4).

To define neurons with activity changes selective for impulsive and omission trials, we computed differences in the $z$ scored spike rate (referenced to baseline; see Materials and Methods) during the delay period between error and correct trial outcomes, as follows: $\mathrm{dZomi}=$ Zomi - Zcor and dZimp $=$ Zimp - Zcor. Neurons were categorized as omission selective if dZomi $<-3$ and impulsive selective if $\mathrm{dZimp}>3$. The results of this classification indicate that 188 of the VM/VAL neurons could be classified as 
A

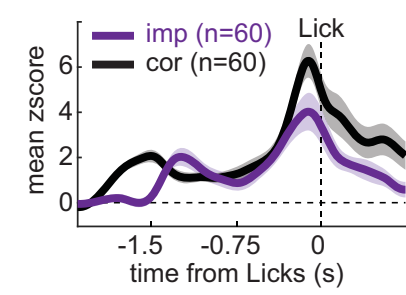

B
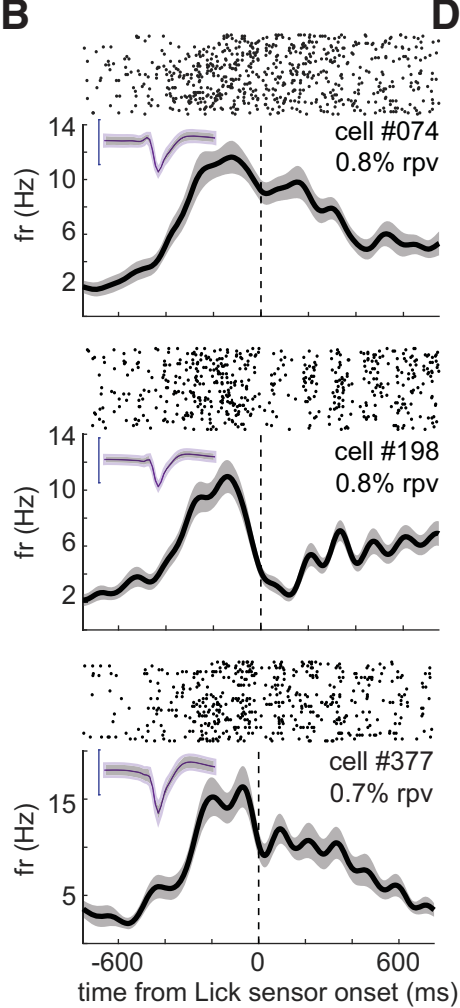

C

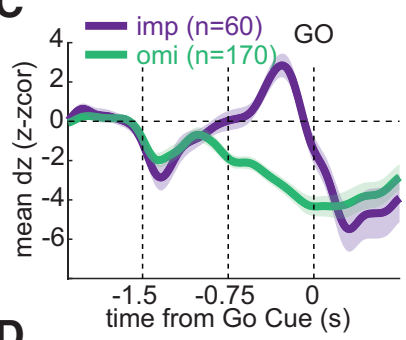

D
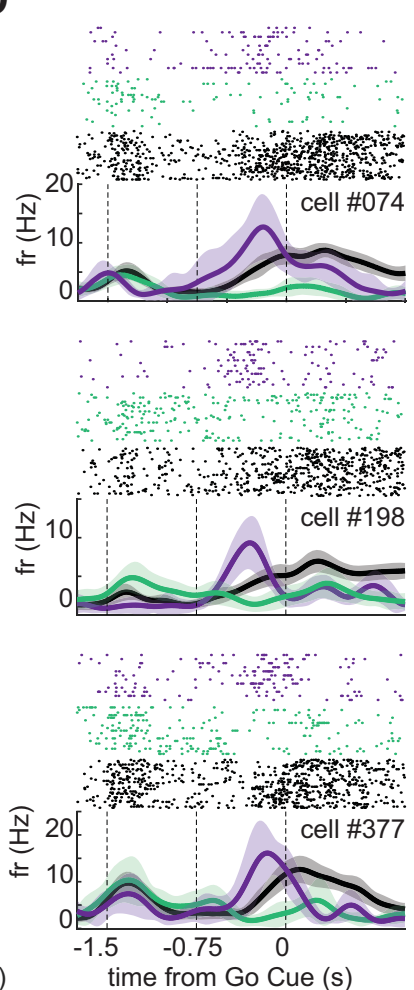

$\mathbf{E}$
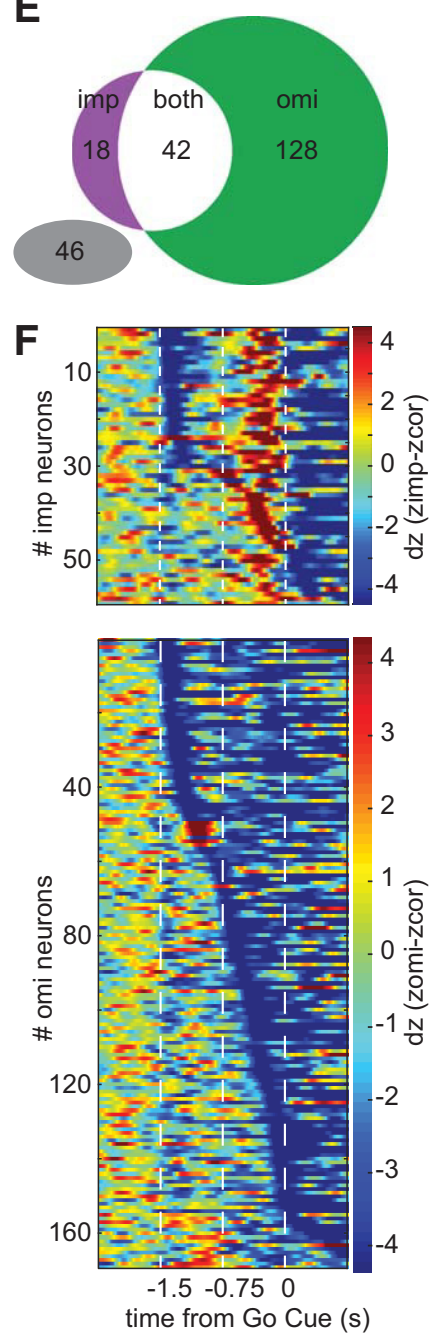

Figure 4. Thalamic ramping activity during the delay is increased before impulsive licking and reduced during omission trials. $\boldsymbol{A}$, Lick-centered population averaged (60 neurons) $z$ score as a function of time for correct and impulsive trials. Note that both trial outcomes show ramping with a similar dynamic up to $600 \mathrm{~ms}$ before the first lick. Shading represents 2 SEMs. $\boldsymbol{B}$, Lick-centered raster and spike rate function from three representative VM/VAL neurons recorded from different mice during correct trials. Violet traces, Average spike wave forms. Each trace is 2 ms in total duration. Calibration: $100 \mu \mathrm{V}$. C, Go cue-centered population-normalized spike rates during imp and omi trials relative to correct trials (dZ; see Materials and Methods). The horizontal dashed lines at $0 \mathrm{dZ}$ indicate no difference from correct trials. Note that during the delay the two populations diverge from 0 in opposite directions, indicating that during the delay impulsive selective neurons tend to overactivate while omi-selective neurons underactivate relative to correct trials. Shading represents 2 SEMs. $\boldsymbol{D}$, Examples of single-unit activity separated by trial type, imp (violet), omi (green), and cor (black). Each panel represents a raster and spike rate function for each type of trial outcome for a representative VM/VAL neuron. Shading in the trial averages represents 2 SEMs. $\boldsymbol{E}$, Venn diagram of $234 \mathrm{VM} / \mathrm{VAL}$ neurons with a significant firing rate difference compared with cor trials during the delay epoch (dZ $>3$ ) for omi trials (green), imp trials (violet), or both (white). No difference (gray). Obtained form 11 sessions, four mice. $\boldsymbol{F}$, Heat map representing $z$ scored activity difference between impulsive and correct trials for all selective units during impulsive trials (top) and for the difference between omission and correct trials (bottom).

omission selective, impulsive selective, or both, with a larger population of omission selectivity (Fig. $4 C, E, F$ ).

To compare the difference in the dynamic changes in neuronal responses between correct and error trials at the population level, we $z$ scored the error trial spike rate function of each neuron with respect to correct trials (Fig. 4C). This analysis revealed a clearly reduced activity in omission-selective neurons during the delay epoch of omission trials. In contrast, impulsive trial-selective neurons showed a steep increase in ramping during the second half of the delay epoch during impulsive trials compared with correct trials. These results obtained at the population level were confirmed at the single-unit level (Fig. 4D). Indeed, raster and spike rate function plots clearly indicated different ramping patterns among the three types of trials (cor, imp, omi), with an early ramping during the impulsive trials and an absence of ramping during the omission trials (Fig. 4D).
Finally, to resolve the timing of imp and omi selectivity at both the single-neuron and population levels; we plotted the $z$ scored rate difference for each neuron as a heat map (Fig. $4 F$ ). For imp-selective neurons during imp trials, a consistent strong increase in spike rate was present during the second half of the delay epoch, while omi-selective neurons during omi trials showed a reduction in activity that could start at varying time points beginning with the stimulus onset, and generally lasted beyond Go-cue presentation. Interestingly, a subpopulation of both omi- and imp-selective neurons showed significantly less activation during the air puff epoch for both types of error trials (Fig. 4C,D,F), suggesting that task-related thalamic neural processing is already altered early in the task when errors occur later.

Overall, these results clearly indicate that the VM/VAL ramping activity is altered during error trials compare with correct trials. Specifically, we provide evidence that the ramping was lacking 
A
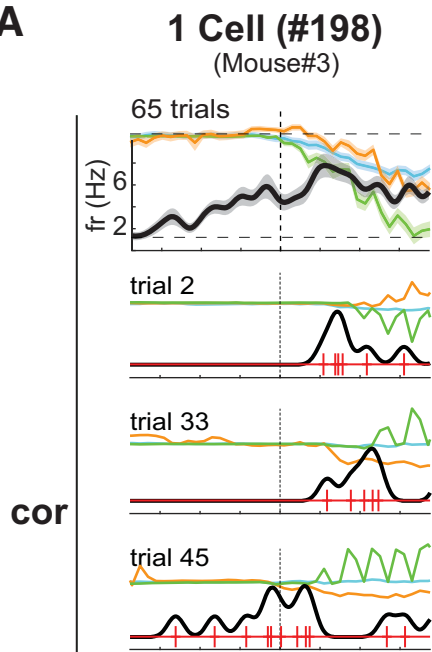

trial 103

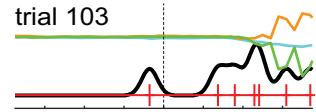

\section{trial 145}
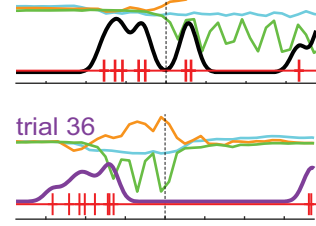

imp

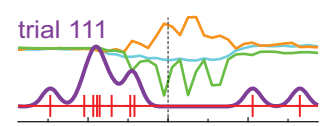

trial 53

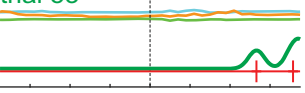

omi

trial 119

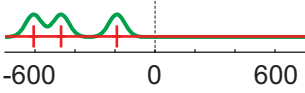

time from GoCue (ms)
1 Cell (\#489)

(Mouse\#5)

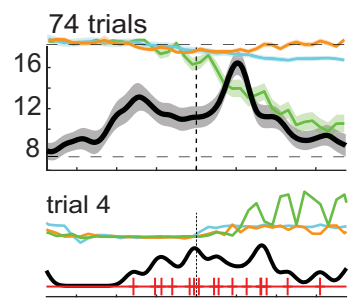

trial 65
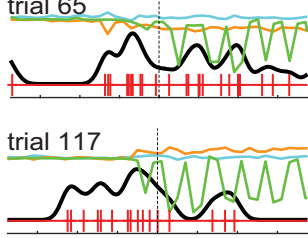

trial 147

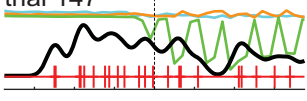

trial 196
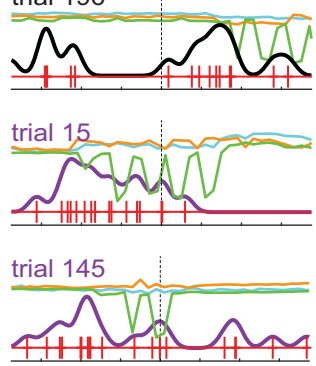

trial 44

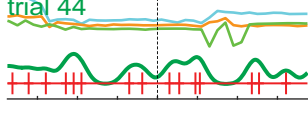

trial 91

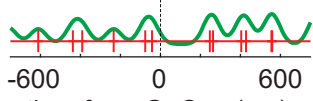

B

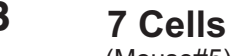

(Mouse\#5)

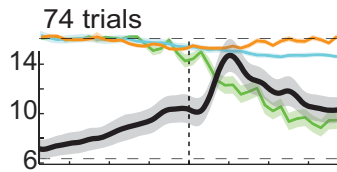

snout

Tongue

PSTH
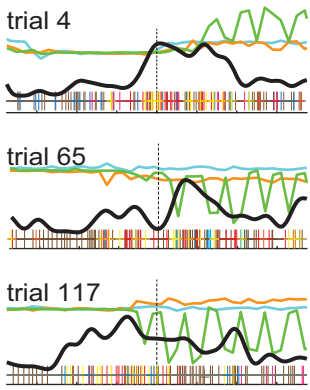

trial 147

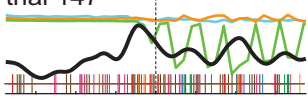

5 tr Average
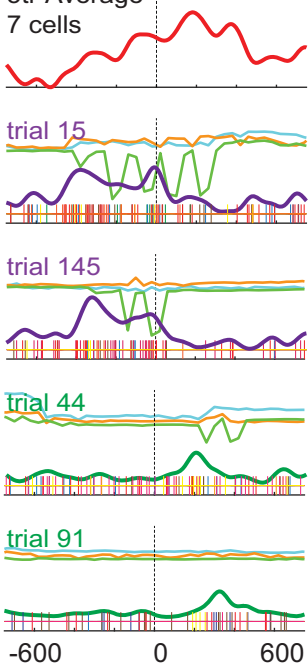

time from GoCue (ms)

Figure 5. Single-trial analysis of motor thalamic neuronal activity in relation to body movements. $A$, Examples of single-trial firing rate changes for two representative VM/VAL neurons (one neuron per column). Spike rate functions (smoothed with a $40 \mathrm{~ms}$ Gaussian kernel) and the corresponding DeepLabCut traces are aligned to the Go cue. The first row shows the average for all correctly executed trials (black trace). The other rows display representative single trials (corresponding to Movies 2, 3), with five cor, two imp, and two omi trials per neuron. Red ticks represent single spikes. Colored traces represent spike rate functions (smoothed with a $40 \mathrm{~ms}$ Gaussian kernel) and the corresponding DeeplabCut traces (video frames were obtained every $40 \mathrm{~ms}$ ). $\boldsymbol{B}$, Same as $\boldsymbol{A}$, but for a group of seven neurons recorded simultaneously (including cell \#489 from $\boldsymbol{A}$ ). Note that the ramping pattern tends to be clearer at the single-trial level for a group of cells compared with a single cell (e.g., trials \#117 and \#147, in $\boldsymbol{A}$ and $\boldsymbol{B}$ ). Vertical ticks denoting spikes emitted by different neurons are identified by color. The sixth row represents the trial av-

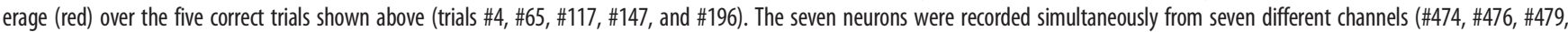
$\# 480, \# 489$, \#492, and \#494; \%rpv: 0.5\%, 1\%,0.9\%, 0.9\%, 0.7\%, 0.9\%, and 0.4\%, respectively).

during omission trials, whereas it peaked too early during the delay of impulsive trials. These results are in support of the hypothesis that ramping is related to the preparation of action release.

\section{Single-trial analysis confirms increased spike rates before lick initiation}

Our analysis so far revealed prominent ramping activity of trialaveraged data for both single neurons and population averages (Figs. 3, 4). However, behavior is controlled on the level of single trials, so the question arises of whether ramping can be observed at a single-trial level for single neurons or for groups of neurons recorded simultaneously. In addition, it is possible that anticipatory neural activity preceding go cues might be highly correlated with ongoing uninstructed movements (Musall et al., 2019). Indeed, if single-trial activity changes were discrete and related to uninstructed movements with progressive likelihood during the delay epoch, this could result in apparent population ramping in trial-averaged results.

To address these questions, we analyzed video recordings of our mice performing the task using DeepLabCut (Nath et al., 2019) to track movements of the tongue, snout, and whiskers for each trial (Fig. 5, Movie 1). For a given trial, we aligned the results of the movement traces with single-unit spiking activity [Fig. $5 \mathrm{~A}$, Movies 2, 3 (corresponding to first and second column, respectively, in Fig. 5A)]. We observed that in all cases (10 cells analyzed from three mice) the spike rate increases seen at the single-trial level were in advance of any detected uninstructed movements 
(often >400 ms; e.g., cell \#189/trial \#145 and cell\#489/trial \#147, both from different mice; Fig. $5 A$ ). However, these spike trains did not have a clear ramping pattern at the single-unit level for single trials, even when the ramping pattern was observed at the level of the trial average (Fig. 5A, top trace).

These results suggest that the time of spike rate change varies from trial to trial and is more discrete than would be expected from a steadily increasing spike rate (i.e., ramp) at the single-trial level. While not all body parts could be tracked with our frontally mounted camera, we note that the single-unit spike rate increases occurred at approximately the same time before lick onset for trials with impulsive early licking (Fig. $5 A$, imp trials) and was markedly reduced in omission trials (Fig. $5 A$, omi trials). These results are fully congruent with the population average in showing a distinct relation to the preparation of lick onset (Fig. $4 A$ ).

To address the question of whether populations of neurons recorded simultaneously show ramping or correlated discrete range changes, we performed a single-trial analysis for a group of seven neurons as an example (Fig. 5B). We observe in our recorded set of neurons that, even at the single-trial level, a ramping pattern becomes discernible as different units start spike rate increases at different times (Fig. 5B). This observation supports the notion that population ramping is a product of variable times of spike rate increase onsets between neurons and constitutes a population-level phenomenon at the single-trial level (see Discussion). To more systematically quantify how much neuron ensemble activity predicts the trial outcome, we use a classifier in the next section.

\section{Thalamic ensemble activity receiving nigral input can be used to accurately predict the behavioral outcome at a single-trial level}

Our preceding analysis of VM/VAL neural activity revealed neuronal preparatory activity before lick onset. We hypothesized that the ramping activity produced at the neuronal population level contained sufficient information to predict each single-trial outcome. To test this hypothesis, we trained a logistic classifier to predict single-trial outcome (e.g., omi vs cor) based on the neuronal activity of randomly chosen subpopulations from 1 to 20 neurons recorded simultaneously (Fig. 6; 100 iterations for each group size $\times 10$ cross-validation). We found that the classifier was able to equally predict trial outcomes for omission versus correct trials (Fig. 6A), impulsive versus correct trials (Fig. 6B), and impulsive versus omission trials (Fig. $6 \mathrm{C}$ ). To control for the possibility that the classifier picked up on the lick activity in imp trials to make its discrimination, we made an additional classification with using spike times only in a $750 \mathrm{~ms}$ window before the decision lick (Fig. $6 B, C$, dotted line). In all cases, we show that the classifier performance was better than chance (dashed line) and improved as a function of the number of neurons used to train it. These results confirm our hypothesis that single-trial outcomes can be predicted by the combined activity of a population of VM/VAL neurons, which tend to produce a ramping pattern, as shown above (Figs. $3-5)$. The more neurons included in the subpopulation, the more accurate was the classifier prediction of single-trial outcome.

\section{Optogenetically suppressing the ramping activity in VM/VAL via the nigro-thalamic pathway reduced impulsive trials and increased omission trials}

The results from the previous sections show that impulsive and omissions trials were associated with a distinct firing pattern in the VM/VAL thalamus during the delay preceding Go cue and presented in trial averages a clear alteration of ramping activity relative to correctly executed trials (Figs. 4,
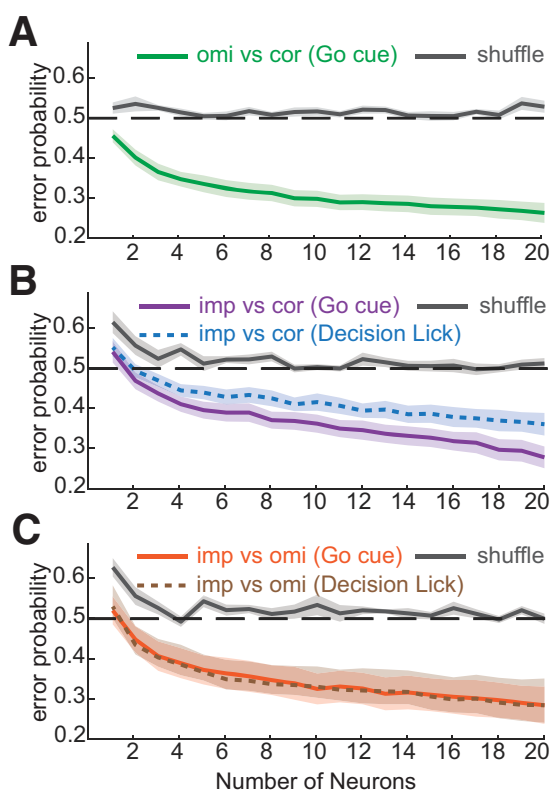

Figure 6. Thalamic population activity during the delay predicts the type of lick timingrelated error trials. $\boldsymbol{A}$, Average performance of logistic classifier on discriminating omission versus correct trials based on the firing rate of VM/VAL neuronal assemblies of different sizes during the 750 ms delay. One hundred iterations for each group size each with a 10-fold cross-validation. Neurons were randomly selected at each iteration. For a shuffle control, trial outcomes were randomly assigned to each trial spike train. The horizontal dashed lines at $50 \%$ indicate the chance level for a binary classification. $\boldsymbol{B}$, Same as $\boldsymbol{A}$ but comparing impulsive and correct trials (solid trace). Because during impulsive trials the mouse licks during the delay, we trained a second classifier that only used the neuronal activity of the 750 ms before the first lick (dotted trace) to exclude the idea that lick-related activity during the delay for imp trials determines the classification. $\boldsymbol{C}$, Same as $\boldsymbol{B}$ but comparing impulsive and omission trials.

5). Using a classifier, these different spike patterns could be used to predict single-trial outcomes (Fig. 6). We next asked whether inhibition from the $\mathrm{SNr}$ during the delay epoch could affect behavioral outcomes through an alteration of ramping activity in VM/VAL.

To optogenetically activate $\mathrm{SNr}$ terminals in VM/VAL, the $\mathrm{SNr}$ of VGAT-Cre (slc32a) mice was injected with an adeno-associated viral vector (rAAV2) containing a Cre-dependent channelrhodopsin 2 (DIO-hChR2-EYFP). SNr terminals expressing ChR2-EYFP (enhanced yellow fluorescent protein) were activated using a blue laser $(473 \mathrm{~nm})$ passing through an optic fiber mounted on a NeuroNexus optrode inserted in VM/VAL thalamic nuclei (Fig. 7A). The $\mathrm{SNr}$ injection site and GFP expression were verified by histology and fluorescence microscopy (Fig. 7B).

During the task, in $20 \%$ of the trials, randomly selected, we stimulated the SNr GABAergic terminals in VM/VAL during the delay epoch ( $1 \mathrm{~s}$ laser pulse starting $250 \mathrm{~ms}$ after the delay epoch onset). We found a significant reduction in the number of impulsive errors during trials with optostimulation in the delay epoch compared with nonopto trials as well as a significant increase in omission error trials (optoON vs optoOFF, two-tailed paired $t$ test for 11 sessions, four mice; pcor $=0.056, p e S i=0.076$, $*$ pimp $=0.030 *$ pomi $=0.025$; Fig. $7 C$ ). In contrast, the SNr stimulation had no effect on the capability of mice to correctly chose left or right lick targets, which is indicated by the errors of licking to the incorrect side remaining equally low (eSi; Fig. 7C). The proportion of correct trials was not significantly changed, likely because a decrease in impulsive trials was offset by an increase in omission trials. 

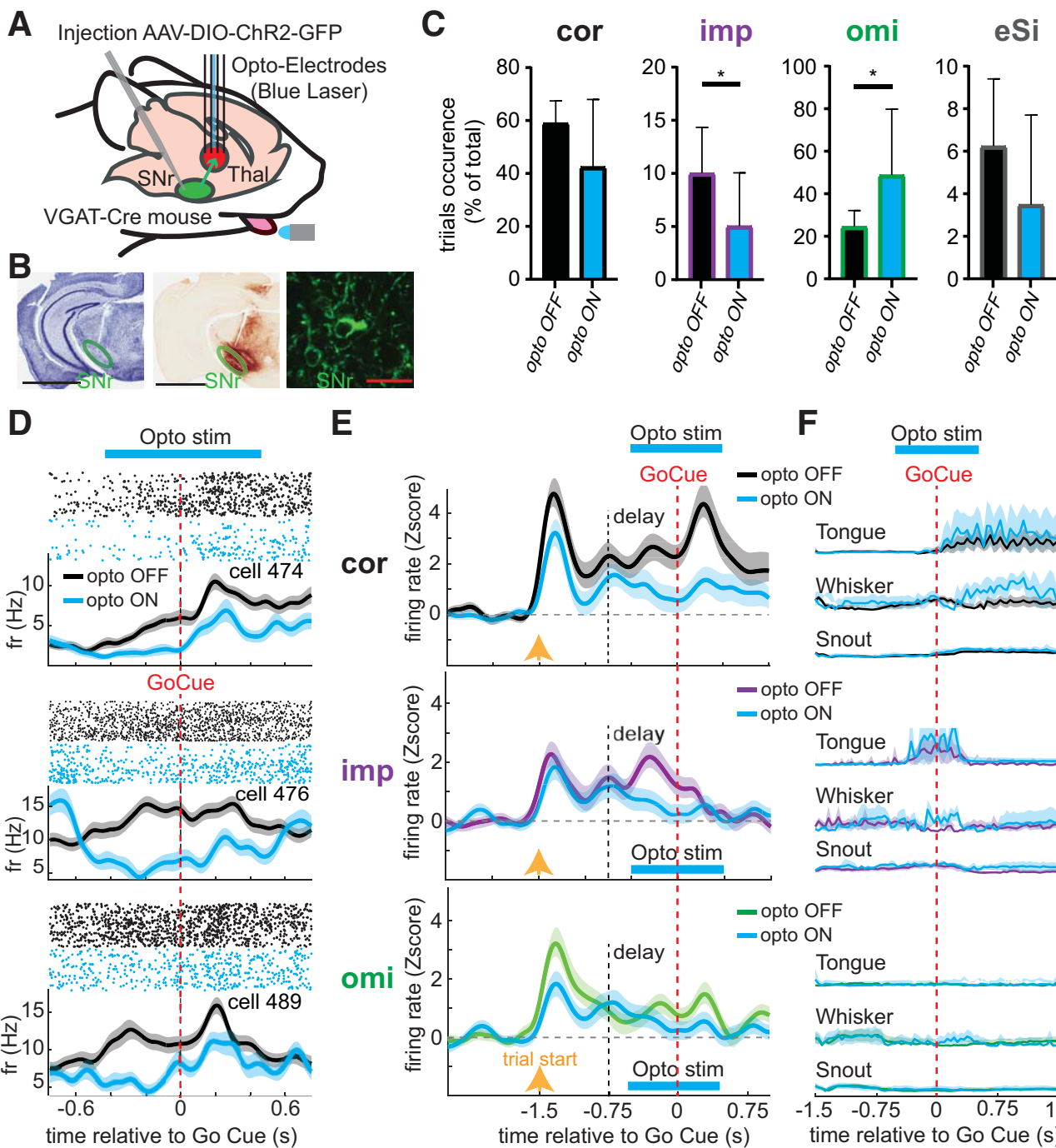

E
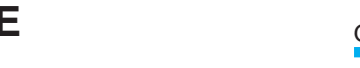

$\mathbf{F}$
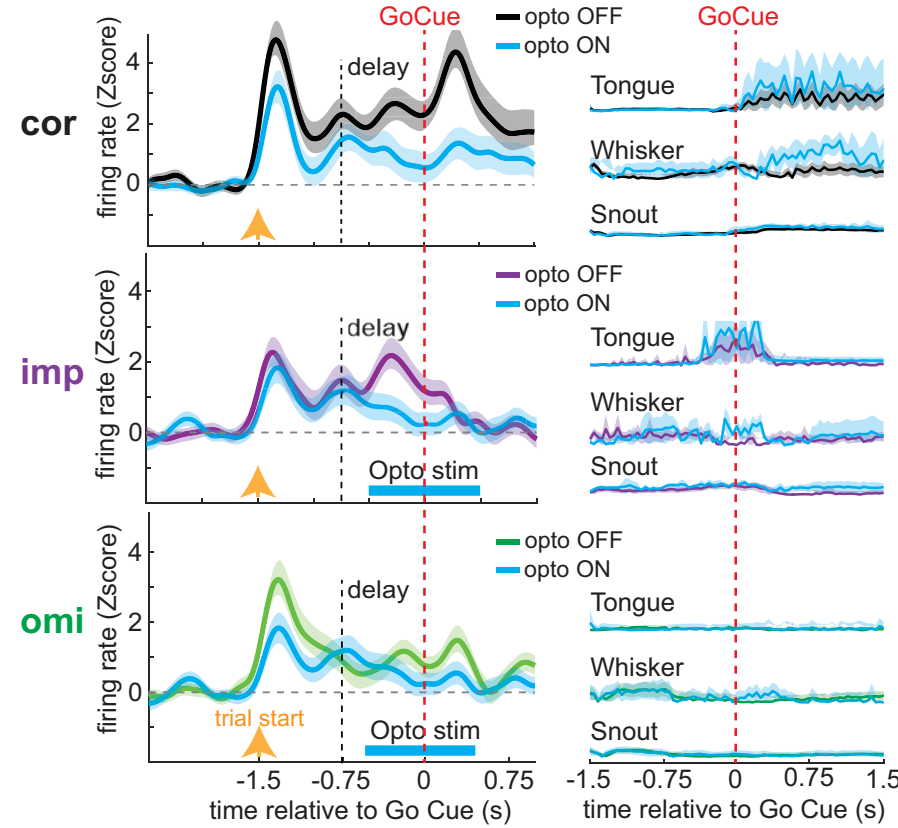

Figure 7. Optogenetically stimulating the nigro-thalamic pathway reduced impulsivity and increased omissions. $A$, Schematic of the experimental paradigm. Slc32a transgenic mice were injected with AAV-DI0-ChR2-EYFP in the SNr to express ChR2. SNr terminals were optogenetically activated by blue light illumination through an optic fiber attached to the NeuroNexus silicon probe located in VM/VAL while the mice performed the task. B. Histology and microscopy confirmed the injection site in the SNr using Nissl staining (left column), immunohistochemistry with anti-GFP revealed with horseradish peroxidase (middle column), and confocal microscopy (right column). Confocal microscopy revealed green fluorescence confirming GFP expression in the somatic membrane of SNr neurons (bottom right). Scale bars: black, $2 \mathrm{~mm}$; red, $20 \mu \mathrm{m}$. C, Behavioral effect of photostimulation. Bar plots representing the probability of occurrence for cor, eSi, imp, and omi trials with laser OFF (black) versus trials with laser ON (cyan; average and SD). D, Raster plots and trial-averaged spike rate functions of three example neurons (rpv 0.5\%, 1\%, $0.7 \%$ from top to bottom) recorded during control correct trials (black) and during optogenetic stimulation trials (cyan). Horizontal blue bars indicate the time of the laser stimulation in trials with optogenetic stimulation. $\boldsymbol{E}$, Averaged z scored firing rate across correct trials with or without photostimulation (0pto stim). Only sessions with at least seven correct opto trials and at least three opto impulsive trials were used ( 89 neurons, five sessions, three mice). The neuronal activity was strongly suppressed by the laser stimulation during the delay epoch (blue) compared with when the laser was OFF (black). Shading represents 2 SEMs. $\boldsymbol{F}$, Video analysis of averaged absolute deviation from baseline for tongue, whisker, and snout movements (DeepLabCut, four sessions, three mice; shading represents 2 SEMs). For each type of trial outcome (cor, imp, omi), the body movements were compared between trials with laser ON versus trials with laser OFF. Note that there was no difference in tracked movements between opto trials and non-opto trials during the delay epoch.

Overall, as a consequence of SNr terminal stimulation during the delay epoch, we observed a significant effect on behavioral outcomes, with a specific effect on error trials related to the timing of action release.

We then asked the question of whether the behavioral effects were mirrored by changes in ramping activity reflecting their differences between correctly executed and impulsive or omission trials. At the single-neuron level, we observed that the laser stimulation tended to reduce the spiking of VM/VAL neurons, as demonstrated in the raster plots and trial-averaged spike rates in Figure $7 D$. It is possible that the optogenetic effect would only be revealed when applied, while the firing rate is rising above the baseline during the task. However, we found that $30 \%$ of 89 tested neurons were significantly inhibited by the laser stimulation outside the task, by performing a statistical analysis on the pretask and post-task optogenetic stimulation (Wilcoxon test, $n$ stim $=54 \pm 30$ laser pulses/session). This control experiment confirms that the optogenetic inhibition of VM/VAL neurons is likely triggered by activating $\mathrm{SNr}$ terminals independently from the task.

The grand average of all VM/VAL thalamic neurons tested ( $n=89$ recorded with a number of impulsive and optostimulation trials sufficient for statistical comparison; Fig. 7E) showed a robust decrease in firing rate during the optogenetic stimulation of nigral terminals, thus preventing the usual ramping from occurring during the delay of the task for all three types of trials (cor, imp, and omi; Fig. 7E). To assess whether laser stimulation resulted in changes of observed movements, we performed a 


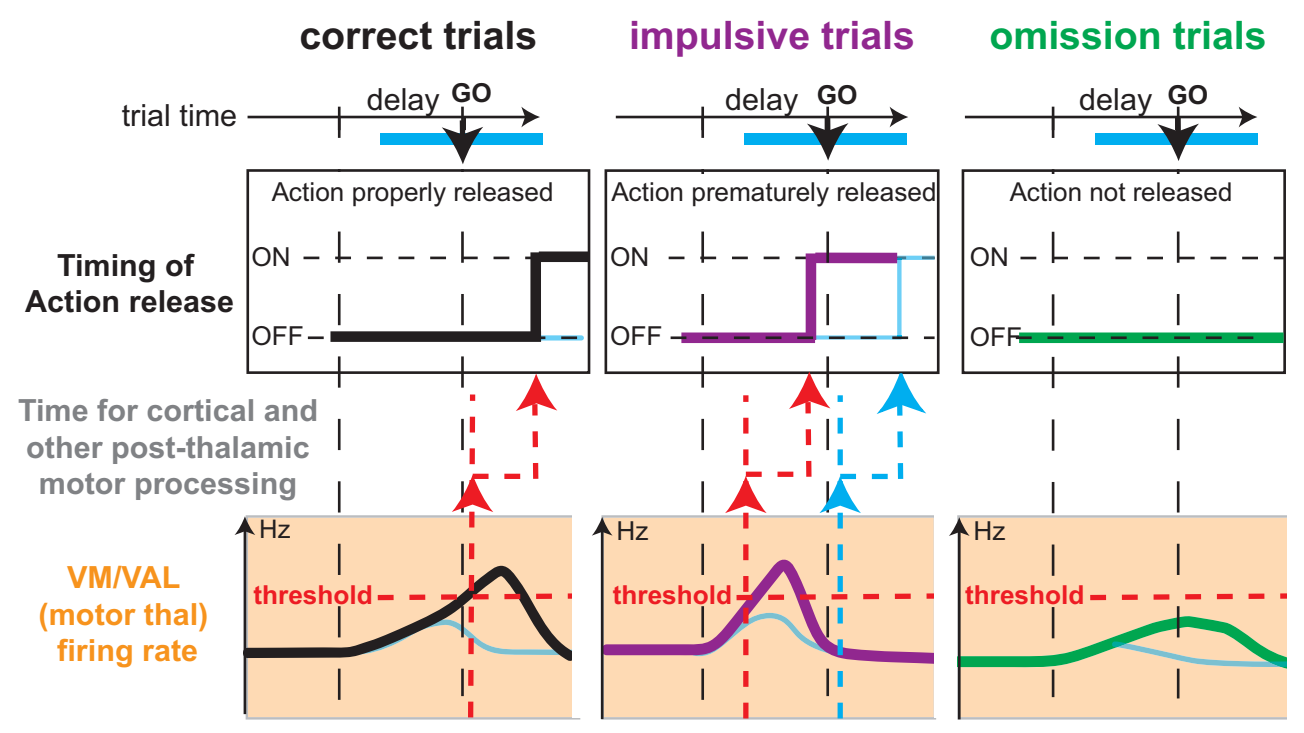

Figure 8. A threshold model for the timing of action release. During correct trials (left column), proper ramping in VM/NAL activity is initiated during the delay and peaks after the Go cue. When a threshold is reached (red), action release within a proper timing window ( $\sim 400 \mathrm{~ms}$ after the Go cue) will be triggered. If the ramping is optogenetically silenced (cyan), the threshold is not reached and the action is not released, resulting in omission trials. During impulsive trials (middle column), the thalamic activity ramps too early and reaches the threshold before the Go cue, leading to an early action release. If the ramping is optogenetically silenced (cyan), the threshold is not reached and the action could be either released post-Go cue, as in a correct trial, or not released at all, as in an omission trial. During omission trials (right column), the thalamic activity will not reach threshold and the action will not be released.

DeepLabCut analysis of movement trajectories for four sessions from three different mice (Fig. $7 F$, Movie 4). No significant changes in movement execution for the three trial outcomes (cor, imp, omi) were observed.

The results of this section demonstrate that nigral terminal stimulation during the delay epoch suppressed VM/VAL ramping, mimicking the diminished or absent ramping associated with spontaneous omissions (Fig. 4). Together, these observations suggest that the VM/VAL pre-motor ramping activity is a necessary part of the proper mechanism of action release and is strongly influenced by nigral input.

\section{Discussion}

\section{Main findings}

In this study, we showed that delay-specific ramping in the VM/ VAL population activity started and peaked before the decision lick in both correct and impulsive trials with similar time courses relative to the lick action release, whereas omission lick trials showed a weak or absent ramping activity. A linear classifier was able to predict the behavioral outcome on a single-trial basis based on the corresponding neuronal ensemble activity from VM/VAL, with greater accuracy for larger neuronal populations. Optogenetic activation of SNr GABAergic terminals in VM/VAL during the delay suppressed ramping activity and resulted in an increase of omissions and a decrease in impulsive licking, supporting a causal impact of nigro-thalamic activity on the timing of action release.

\section{Ramping in VM/VAL population anticipates action release}

The population average of VM/VAL neurons in our data ramped and peaked at $\sim 650$ and $\sim 160 \mathrm{~ms}$, respectively, before the tongue touched the spout. Given that $\sim 33 \mathrm{~ms}$ of tongue protrusion for a mouse cue elicited licks before touching the lick tube (Bollu et al., 2019), the peak in ramping clearly preceded movement initiation, and in most trials we found no other preceding tongue, nose, or whisker movement. Given previous findings that VM/VAL neurons project directly to ALM premotor cortex (Guo et al., 2018), it is plausible that thalamocortical neurons reaching a specific firing rate threshold could be an important link in a sequence of neuronal cortical activations that will ultimately lead to lick action release.

In primates, a similar relationship to saccadic movement release has previously been observed in VA/VL motor thalamus, where ramping activity was found before cued delayed or selfinitiated saccades, and such ramping was hypothesized to derive from basal ganglia signals (Tanaka, 2007). Interestingly, in premotor cortex of primates, the peak in ramping activity was observed $\sim 280 \mathrm{~ms}$ before action initiation (Thura and Cisek, 2014), similar to the timing observed in our study. In addition, ramping in frontal cortical circuits before movement has been linked to an interval timing mechanism (Narayanan, 2016).

\section{A threshold model for timing of movement initiation}

Together, our electrophysiological and optogenetic results support a parsimonious model of how the nigro-thalamic pathway might be involved in the neuronal processes related to action release (Fig. 8). In this model, movement will be initiated only if a threshold in the ramping of VM/VAL population activity is reached (Fig. 8, correct trials). If this ramping reaches the threshold too early, it will trigger a premature action (Fig. 8, impulsive trials). In contrast, if it never reaches the threshold, the prepared action will not occur (Fig. 8, omission trials).

In the context of the proposed threshold model, we posit that by default the level of activity in the motor thalamus would be maintained at subthreshold level by tonic GABAergic input from the SNr (Delong, 1971; Ruskin et al., 2002; Lobb and Jaeger, 2015). Reduction of tonic SNr inhibition may provide a signal for VM/VAL activity to ramp up toward the action threshold. This hypothesis is similar to the classic function of SNr inhibition to trigger saccades in primates (Wurtz and Hikosaka, 1986) and the urgency function ascribed to GPi downramping before delayed reaching movement onset (Thura and Cisek, 2017). In case of a sudden action stop signal (countermanding), the $\mathrm{SNr}$ can suddenly reincrease its activity and prevent the action to be released (Schmidt et al., 2013). Our optogenetic results fully 
support this stopping function for basal ganglia output, as optogenetic activation in the delay epoch leads to a decrease in impulsive and increase in omission error trials.

\section{The basal ganglia output is in a key position to influence ramping}

While our optogenetic manipulation focused on the final output of the basal ganglia to motor thalamus, previous work has shown similar ramping activity in relation to movement initiation in preceding stages of basal ganglia processing. In striatum, ramping has been seen during cued preparatory delays (Jaeger et al., 1993; Mello et al., 2015; Emmons et al., 2017) and before self-initiated movements (Lee and Assad, 2003). In GPe and GPi in primates, ramping was ascribed to a "motor-readiness" (Jaeger et al., 1993), functionally identical to an "urgency" function (Thura and Cisek, 2017). Neurons with downramps of activity were described in primate GPi before delayed reaching movement onset (Thura and Cisek, 2017), which supported the proposition in our model that the basal ganglia control upramping in VM/ VAL. In contrast, subthalamic excitation of the nigra may favor the stopping of movement initiation (Schmidt et al., 2013). In agreement with this notion, optogenetic inhibition of the direct pathway (disinhibiting $\mathrm{SNr}$ ) leads to a delay in the time of motor execution (Tecuapetla et al., 2016). While the original source for such ramping is hard to pinpoint, it may well be related to ramping activity in dopamine neurons that project to the striatum (BG input nucleus) before rewarded actions (Howe et al., 2013; Lloyd and Dayan, 2015).

\section{VM/VAL pre-motor ramping relates to vigor, urgency, or timing of action release}

The hypothesis that cortical ramping activity represents increasing urgency for commitment to the action was proposed by Cisek et al. (2009) in a sequence of studies in primates (Thura et al., 2012, 2014).

The vigor hypothesis posits that BG output controls movement vigor as measured by the impact of basal ganglia output on movement speed (Desmurget and Turner, 2008; Turner and Desmurget, 2010). This effect has also been referred to as a control of "movement gain" (Anderson and Horak, 1985), which may be related to motivation (Catanese and van der Meer, 2013; McGinty et al., 2013) and cost of action (Shadmehr and Krakauer, 2008; Turner and Desmurget, 2010). Clinically, movement gain is diminished in hypometric basal ganglia disorders including Parkinson's disease, often leading to bradykinesia and hypometria (Desmurget et al., 2004).

In the frame of the threshold hypothesis model presented in Figure 8, the activity level in VM/VAL could be a function of the gain (vigor, urgency) for a given action: undervigorous (omission) and overvigorous (impulsive) actions. This gain function combined with an adjustable threshold value could serve as a timer for action release, as well as a system for selection based on activity level originating in the direct and indirect pathways of striatum (Klaus et al., 2019). Indeed, it is possible that other actions compete (Leblois et al., 2006; Boraud et al., 2018) for the highest urgency value, and that a winner-take-all mechanism is implemented. Such a possibility is also compatible with our proposed mechanism, in which the first action to reach the threshold would be released.

\section{Single-trial activity points to population coding of ramping activity}

Our single-trial analysis (Fig. 6) suggests that individual neurons show relatively discrete rate increases during the delay epoch, but that different neurons within the same trial have different change points in firing rate, leading to the population-averaged spiking activity to ramp even for a single trial. A population code for ramping in the prediction of movement timing is further supported by the ability of our linear decoder to correctly decipher trial outcomes with incremental quality for increasing population size. Overall, our data suggest that a basal ganglia urgency output may provide a moving set point of a discrete attractor model (Inagaki et al., 2019). VM/VAL thalamic neurons have been found to be in an excitatory feedback loop with ALM premotor cortex for mouse lick decision-making (Guo et al., 2017). A competing attractor for right and left decisions is likely presented by different neural populations both at the level of VM/VAL thalamus and ALM cortex, but both populations may be driven by the basal ganglia urgency downramp for delayed behavioral execution with an expected time of action release. Future work is still needed, however, to clearly delineate whether basal ganglia output to motor thalamus also multiplexes action-specific signals with such an urgency ramp, as would be expected from the frequently hypothesized function of basal ganglia in action selection (Humphries and Gurney, 2002; Bogacz and Larsen, 2011).

\section{An interpretation of complex VM/VAL task-specific responses}

In addition to ramping during the delay, VM/VAL tended to be strongly activated shortly after the onset of the air-puff epoch (Fig. 3F). Such activity could be associated with stimulus encoding, but also with higher-order cognitive components of task processing such as time estimation (Emmons et al., 2017) or reward prediction mechanisms (Sato and Hikosaka, 2002). Complex responses are also well known for both of the major input structures to VM/VAL, namely premotor cortex and the SNr. Indeed, early work by Wurtz and Hikosaka (1986) discriminated 10 response types in primate $\mathrm{SNr}$ neurons that appeared to be both cognitive and sensory in nature. Such constant modulation of nigral inhibition therefore is likely to sculpt an equally complex time course in strongly connected structures, such as VM/VAL in mice. Again, additional studies are needed to disentangle the function of each neural response type with respect to task execution in mouse decision-making.

\section{References}

Alexander G, Crutcher M (1990) Functional architecture of basal ganglia circuits: neural substrates of parallel processing. Trends Neurosci 13:266-271.

Anderson ME, Horak FB (1985) Influence of the globus pallidus on arm movements in monkeys. III. Timing of movement-related information. J Neurophysiol 54:433-448.

Bodor AL, Giber K, Rovó Z, Ulbert I, Acsády L (2008) Structural correlates of efficient GABAergic transmission in the basal ganglia-thalamus pathway. J Neurosci 28:3090-3102.

Bogacz R, Larsen T (2011) Integration of reinforcement learning and optimal decision-making theories of the basal ganglia. Neural Comput 23:817-851.

Bollu TP, Whitehead S, Kardon B, Redd J, Liu MH, Goldberg J (2019) Tongue kinematics. Cortex-dependent corrections as the mouse tongue reaches for, and misses, targets. bioRxiv. doi: https://doi.org/10.1101/ 655852.

Boraud T, Leblois A, Rougier NP (2018) A natural history of skills. Prog Neurobiol 171:114-124.

Bosch-Bouju C, Hyland BI, Parr-Brownlie LC (2013) Motor thalamus integration of cortical, cerebellar and basal ganglia information: implications for normal and parkinsonian conditions. Front Comput Neurosci 7:163.

Buhusi CV, Meck WH (2005) What makes us tick? Functional and neural mechanisms of interval timing. Nat Rev Neurosci 6:755-765.

Catanese J, van der Meer M (2013) A network state linking motivation and action in the nucleus accumbens. Neuron 78:753-754. 
Cisek P, Puskas GA, El-Murr S (2009) Decisions in changing conditions: the urgency-gating model. J Neurosci 29:11560-11571.

Delong MR (1971) Activity of pallidal neurons during movement. J Neurophysiol 34:414-427.

Desmurget M, Turner RS (2008) Testing basal ganglia motor functions through reversible inactivations in the posterior internal globus pallidus. J Neurophysiol 99:1057-1076.

Desmurget M, Grafton ST, Vindras P, Gréa H, Turner RS (2004) The basal ganglia network mediates the planning of movement amplitude. Eur J Neurosci 19:2871-2880.

Donahue CH, Kreitzer AC (2015) A direct path to action initiation. Neuron 88:240-241.

Emmons XEB, Corte BJ, De Kim Y, Parker KL, Matell XS, Narayanan NS (2017) Rodent medial frontal control of temporal processing in the dorsomedial striatum. J Neurosci 37:8718-8733.

Graybiel AM (2008) Habits, rituals, and the evaluative brain. Annu Rev Neurosci 31:359-387.

Guo K, Yamawaki N, Svoboda K, Shepherd GMG (2018) Anterolateral motor cortex connects with a medial subdivision of ventromedial thalamus through cell type-specific circuits, forming an excitatory thalamocortico-thalamic loop via layer 1 apical tuft dendrites of layer 5B pyramidal tract type neurons. J Neurosci 38:8787-8797.

Guo ZV, Hires SA, Li N, O'Connor DH, Komiyama T, Ophir E, Huber D, Bonardi C, Morandell K, Gutnisky D, Peron S, Xu N-1, Cox J, Svoboda K (2014a) Procedures for behavioral experiments in head-fixed mice. PLoS One 9:e88678.

Guo ZV, Li N, Huber D, Ophir E, Gutnisky D, Ting JT, Feng GP, Svoboda K (2014b) Flow of cortical activity underlying a tactile decision in mice. Neuron 81:179-194.

Guo ZV, Inagaki HK, Daie K, Druckmann S, Gerfen CR, Svoboda K (2017) Maintenance of persistent activity in a frontal thalamocortical loop. Nature 545:181-186.

Howe MW, Tierney PL, Sandberg SG, Phillips PEM, Graybiel AM (2013) Prolonged dopamine signalling in striatum signals proximity and value of distant rewards. Nature 500:575-579.

Humphries MD, Gurney KN (2002) The role of intra-thalamic and thalamocortical circuits in action selection. Netw Comput Neural Syst 13:131156.

Inagaki HK, Fontolan L, Romani S, Svoboda K (2019) Discrete attractor dynamics underlies persistent activity in the frontal cortex. Nature 566:212217.

Jaeger D, Gilman S, Aldridge JW (1993) Primate basal ganglia activity in a precued reaching task: preparation for movement. Exp Brain Res 95:5164.

Jaeger D, Gilman S, Aldridge JW (1995) Neuronal activity in the striatum and pallidum of primates related to the execution of externally cued reaching movements. Brain Res 694:111-127.

Klaus A, Alves da Silva J, Costa RM (2019) What, if, and when to move: basal ganglia circuits and self-paced action initiation. Annu Rev Neurosci 42:459-483.

Kuramoto E, Fujiyama F, Nakamura KC, Tanaka Y, Hioki H, Kaneko T (2011) Complementary distribution of glutamatergic cerebellar and GABAergic basal ganglia afferents to the rat motor thalamic nuclei. Eur J Neurosci 33:95-109.

Leblois A, Boraud T, Meissner W, Bergman H, Hansel D (2006) Competition between feedback loops underlies normal and pathological dynamics in the basal ganglia. J Neurosci 26:3567-3583.

Lee IH, Assad JA (2003) Putaminal activity for simple reactions or self-timed movements. J Neurophysiol 89:2528-2537.

Li N, Chen TW, Guo ZV, Gerfen CR, Svoboda K (2015) A motor cortex circuit for motor planning and movement. Nature 519:51-56.

Lloyd K, Dayan P (2015) Tamping ramping: algorithmic, implementational, and computational explanations of phasic dopamine signals in the accumbens. PLoS Comput Biol 11:e1004622.

Lobb CJ, Jaeger D (2015) Bursting activity of substantia nigra pars reticulata neurons in mouse parkinsonism in awake and anesthetized states. Neurobiol Dis 75:177-185.
McGinty VB, Lardeux S, Taha SA, Kim JJ, Nicola SM (2013) Invigoration of reward seeking by cue and proximity encoding in the nucleus accumbens. Neuron 78:910-922.

Mello GBM, Soares S, Paton JJ (2015) A scalable population code for time in the striatum. Curr Biol 25:1113-1122.

Mink JW (1996) BG competing action selection model. Prog Neurobiol 50:381-425.

Mirzaei XA, Kumar XA, Leventhal XD, Mallet XN, Aertsen XA, Berke J, Schmidt XR (2017) Sensorimotor processing in the basal ganglia leads to transient beta oscillations during behavior. J Neurosci 37:11220-11232.

Musall S, Kaufman MT, Juavinett AL, Gluf S, Churchland AK (2019) Singletrial neural dynamics are dominated by richly varied movements. Nat Neurosci 22:1677-1686

Narayanan NS (2016) Ramping activity is a cortical mechanism of temporal control of action. Curr Opin Behav Sci 8:226-230.

Nath T, Mathis A, Chen AC, Patel A, Bethge M, Mathis MW (2019) Using DeepLabCut for 3D markerless pose estimation across species and behaviors. Nat Protoc 14:2152-2176.

Quiroga RQ (2012) Spike sorting. Curr Biol 22:R45-R46.

Quiroga RQ, Nadasdy Z, Ben-Shaul Y (2004) Unsupervised spike detection and sorting with wavelets and superparamagnetic clustering. Neural Comput 16:1661-1687.

Ruskin DN, Bergstrom DA, Walters JR (2002) Nigrostriatal lesion and dopamine agonists affect firing patterns of rodent entopeduncular nucleus neurons. J Neurophysiol 88:487-496.

Sakai ST, Grofova I, Bruce K (1998) Nigrothalamic projections and nigrothalamocortical pathway to the medial agranular cortex in the rat: singleand double-labeling light and electron microscopic studies. J Comp Neurol 391:506-525.

Sato M, Hikosaka O (2002) Role of primate substantia nigra pars reticulata in reward-oriented saccadic eye movement. J Neurosci 22:2363-2373.

Schmidt R, Leventhal DK, Mallet N, Chen F, Berke JD (2013) Canceling actions involves a race between basal ganglia pathways. Nat Neurosci 16:1118-1124.

Shadmehr R, Krakauer JW (2008) A computational neuroanatomy for motor control. Exp Brain Res 185:359-381.

Shamash P, Carandini M, Harris K, Steinmetz N (2018) A tool for analyzing electrode tracks from slice histology. bioRxiv. doi: https://doi.org/ $10.1101 / 447995$

Sukiban J, Voges N, Dembek TA, Pauli R, Visser-Vandewalle V, Denker M, Weber I, Timmermann L, Grün S (2019) Evaluation of spike sorting algorithms: application to human subthalamic nucleus recordings and simulations. Neuroscience 414:168-185.

Tanaka M (2007) Cognitive signals in the primate motor thalamus predict saccade timing. J Neurosci 27:12109-12118.

Tecuapetla F, Jin X, Lima SQ, Costa RM (2016) Complementary contributions of striatal projection pathways to action initiation and execution. Cell 166:703-715.

Theyel BB, Llano DA, Sherman SM (2010) The corticothalamocortical circuit drives higher-order cortex in the mouse. Nat Neurosci 13:84-88.

Thura D, Cisek P (2014) Deliberation and commitment in the premotor and primary motor cortex during dynamic decision making. Neuron 81:1401-1416.

Thura D, Cisek P (2017) The basal ganglia do not select reach targets but control the urgency of commitment. Neuron 95:1160-1170.

Thura D, Beauregard-Racine J, Fradet CW, Cisek P (2012) Decision making by urgency gating: theory and experimental support. J Neurophysiol 108:2912-2930.

Thura D, Cos I, Trung J, Cisek P (2014) Context-dependent urgency influences speed-accuracy trade-offs in decision-making and movement execution. J Neurosci 34:16442-16454

Turner RS, Desmurget M (2010) Basal ganglia contributions to motor control: a vigorous tutor. Curr Opin Neurobiol 20:704-716.

Vong L, Ye C, Yang Z, Choi B, Chua S, Lowell BB (2011) Leptin Action on GABAergic Neurons Prevents Obesity and Reduces Inhibitory Tone to POMC Neurons. Neuron 71:142-154.

Wurtz RH, Hikosaka O (1986) Role of the basal ganglia in the initiation of saccadic eye movements. Prog Brain Res 64:175-190. 